JOURNAL OF THE

AMERICAN MATHEMATICAL SOCIETY

Volume 14, Number 2, Pages 429-469

S 0894-0347(00)00360-X

Article electronically published on December 8, 2000

\title{
DEFINABLE SETS, MOTIVES AND $p$-ADIC INTEGRALS
}

\author{
JAN DENEF AND FRANÇOIS LOESER
}

\section{INTRODUCTION}

0.1. Let $X$ be a scheme, reduced and separated, of finite type over $\mathbf{Z}$. For $p$ a prime number, one may consider the set $X\left(\mathbf{Z}_{p}\right)$ of its $\mathbf{Z}_{p}$-rational points. For every $n$ in $\mathbf{N}$, there is a natural map $\pi_{n}: X\left(\mathbf{Z}_{p}\right) \rightarrow X\left(\mathbf{Z} / p^{n+1}\right)$ assigning to a $\mathbf{Z}_{p}$-rational point its class modulo $p^{n+1}$. The image $Y_{n, p}$ of $X\left(\mathbf{Z}_{p}\right)$ by $\pi_{n}$ is exactly the set of $\mathbf{Z} / p^{n+1}$-rational points which can be lifted to $\mathbf{Z}_{p}$-rational points. Denote by $N_{n, p}$ the cardinality of the finite set $Y_{n, p}$. By a result of the first author [7], the Poincaré series

$$
P_{p}(T):=\sum_{n \in \mathbf{N}} N_{n, p} T^{n}
$$

is a rational function of $T$. Later Macintyre [24], Pas 26] and Denef [10] proved that the degrees of the numerator and denominator of the rational function $P_{p}(T)$ are bounded independently of $p$. One task of the present paper is to prove a much stronger uniformity result by constructing a canonical rational function $P_{\text {ar }}(T)$ which specializes to $P_{p}(T)$ for almost all $p$. It follows in particular from our results that there exist, for every $n$ in $\mathbf{N}$, varieties $Z_{n, i}$ and rational numbers $r_{n, i}$ in $\mathbf{Q}$, $1 \leq i \leq m_{n}$, such that, for almost all $p$ and every $n$,

$$
N_{n, p}=\sum_{1 \leq i \leq m_{n}} r_{n, i} \operatorname{card} Z_{n, i}\left(\mathbf{F}_{p}\right)
$$

Hence a natural idea would be to try to construct $P_{\operatorname{ar}}(T)$ as a series with coefficients in $K_{0}\left(\mathrm{Sch}_{\mathbf{Q}}\right) \otimes \mathbf{Q}$, with $K_{0}\left(\mathrm{Sch}_{\mathbf{Q}}\right)$ the "Grothendieck ring of algebraic varieties over Q", defined in 1.2 . However, since different varieties over a number field may have the same $L$-function, and we want the function $P_{\text {ar }}(T)$ to be canonical, we have to replace the varieties $Z_{n, i}$ by Chow motives and the naive Grothendieck ring $K_{0}\left(\operatorname{Sch}_{\mathbf{Q}}\right) \otimes \mathbf{Q}$ by the ring $K_{0}^{v}\left(\operatorname{Mot}_{\mathbf{Q}, \overline{\mathbf{Q}}}\right) \otimes \mathbf{Q}$, with $K_{0}^{v}\left(\operatorname{Mot}_{\mathbf{Q}, \overline{\mathbf{Q}}}\right)$ the image of $K_{0}\left(\mathrm{Sch}_{\mathbf{Q}}\right)$ in the Grothendieck ring of Chow motives with coefficients in $\overline{\mathbf{Q}}$, as defined in 1.3

We can now state our uniformity result on the series $P_{p}(T)$ as follows.

0.2. Theorem. Given $X$ as above, there exists a canonical series $P_{\text {ar }}(T)$ with coefficients in $K_{0}^{v}\left(\operatorname{Mot}_{\mathbf{Q}, \overline{\mathbf{Q}}}\right) \otimes \mathbf{Q}$, which is a rational function of $T$ and which specializes - after taking the trace of the Frobenius on an étale realization - onto the p-adic Poincaré series $P_{p}(T)$, for almost all prime numbers $p$.

Received by the editors October 6, 1999 and, in revised form, October 20, 2000.

2000 Mathematics Subject Classification. Primary 03C10, 03C98, 12E30, 12L12, 14G15, 14G20, 14G27; Secondary 11G25, 11S40, 12L10, 14F20, 14G05, 14G10, 14J20. 
In fact, one constructs in 9.2 for $X$ a variety over any field $k$ of characteristic zero, a canonical series $P_{\mathrm{ar}}(T)$ with coefficients in the Grothendieck ring $K_{0}^{v}\left(\operatorname{Mot}_{k, \overline{\mathbf{Q}}}\right) \otimes \mathbf{Q}$ - defined in 1.3 - which is equal to the former one when $k=\mathbf{Q}$ and, furthermore, the series $P_{\mathrm{ar}}(T)$ is rational in a precise sense (Theorem [9.2.1).

0.3. Now assume $X$ is a subscheme of the affine space $\mathbf{A}_{\mathbf{Z}}^{m}$ defined by $f_{1}=\ldots=$ $f_{r}=0$, with $f_{1}, \ldots, f_{r}$ in $\mathbf{Z}\left[x_{1}, \ldots, x_{m}\right]$. The starting point in the proof of the rationality of the series $P_{p}(T)$ in [7] is the relation $P_{p}\left(p^{-m-1} p^{-s}\right)=\frac{p}{p-1} I_{p}(s)$, with

$$
I_{p}(s)=\int_{W_{p}}|w|_{p}^{s}|d x|_{p}|d w|_{p}
$$

where $W_{p}$ is defined as

$$
\begin{aligned}
W_{p}:=\left\{(x, w) \in \mathbf{Z}_{p}^{m} \times \mathbf{Z}_{p} \mid\right. \\
\left.\quad \exists y \in \mathbf{Z}_{p}^{m}: x \equiv y \quad \bmod w, \text { and } f_{i}(y)=0, \text { for } i=1, \ldots, r\right\} .
\end{aligned}
$$

In particular, $W_{p}$ is defined by a formula in the first order language of valued fields independently of the prime $p$.

More generally, let $k$ be a finite extension of $\mathbf{Q}$ with ring of integers $\mathcal{O}$ and $R=\mathcal{O}\left[\frac{1}{N}\right]$, with $N$ a nonzero multiple of the discriminant. For $x$ a closed point of Spec $R$, we denote by $\mathcal{O}_{x}$ the completion of the localization of $R$ at $x$, by $K_{x}$ its fraction field, and by $\mathbf{F}_{x}$ the residue field at $x$, a finite field of cardinality $q_{x}$. Let $f(x)$ be a polynomial in $k\left[x_{1}, \ldots, x_{m}\right]$ (or more generally a definable function in the first order language of valued fields with variables and values taking place in the valued field and with coefficients in $k$ ) and let $\varphi$ be a formula in the language of valued fields with coefficients in $k$, free variables $x_{1}, \ldots, x_{m}$ running over the valued field and no other free variables. Now set $W_{x}:=\left\{y \in \mathcal{O}_{x}^{m} \mid \varphi(y)\right.$ holds in $\left.K_{x}\right\}$ and define

$$
I_{\varphi, f, x}(s)=\int_{W_{x}}|f(y)|_{x}^{s}|d y|_{x},
$$

for $x$ a closed point of $\operatorname{Spec} \mathcal{O}$, with similar notation as before. In Theorem 8.5.1 we show more generally that there exists a canonical rational function $I_{\varphi, f, \operatorname{mot}}(T)$ with coefficients in an appropriate Grothendieck ring of motives, such that, for almost all closed points $x$ in $\operatorname{Spec} \mathcal{O}, I_{\varphi, f, \operatorname{mot}}(T)$ specializes - after taking the trace of the Frobenius on an étale realization and after setting $T=q_{x}^{-s}$ - to the $p$-adic integral $I_{\varphi, f, x}(s)$.

0.4. Let $k$ be a field of characteristic zero. In section 3 to every formula $\varphi$ in the first order language of rings with coefficients in $k$ we associate, in a canonical way, a virtual motive $\chi_{c}(\varphi)$ in the ring $K_{0}^{v}\left(\operatorname{Mot}_{k, \overline{\mathbf{Q}}}\right) \otimes \mathbf{Q}$. This virtual motive $\chi_{c}(\varphi)$ depends only on the sets defined by $\varphi$ over the pseudo-finite fields containing $k$. When $k$ is of finite type over $\mathbf{Q}$, one recovers the number of points of the sets defined by $\varphi$ over the residual fields at almost all finite places of $k$ by taking the trace of the corresponding Frobenius on an étale realization of $\chi_{c}(\varphi)$. It is quite amazing to note that, since virtual motives have Euler characteristic and Hodge numbers (with compact supports), it follows from our results that every formula with coefficients in $k$ has an Euler characteristic in $\mathbf{Q}$ and also Hodge numbers in $\mathbf{Q}$. One of the simplest examples is the formula $(\exists y) x=y^{n}$ which has Euler characteristic $\frac{1}{n}$ and Hodge polynomial $\frac{1}{n} u v$. 
0.5. The construction in section 3 relies on the work of Fried, Haran, Jarden and Sacerdote on Galois stratifications and quantifier elimination for pseudo-finite fields [19], 16], 18. More precisely, quantifier elimination for pseudo-finite fields allows us to replace our original formula by a quantifier free Galois formula, i.e. a quantifier free formula in the language of rings and constants in $k$, extended by Artin symbols $\operatorname{Ar}_{W / U}(x)$ corresponding to the decomposition subgroup of $\mathrm{Gal}(W / U)$ at $x$ modulo conjugation, with $x$ a variable running over the rational points of $U$. Here $W$ is an étale Galois cover of a normal algebraic variety $U$. Then, by using Artin's theorem on induced representation - a tool already present in the work of Fried and Jarden [18] - and also recent results on motives [20, [21, [2, we are able to associate a canonical virtual motive to every quantifier free Galois formula.

0.6. In a previous paper 12, we developed a general theory of integration - called motivic integration - on the space of arcs of an algebraic variety $X$. More precisely, let $X$ be an algebraic variety over a field $k$ of characteristic zero. The space of $\operatorname{arcs} \mathcal{L}(X)$ is a $k$-scheme whose $K$-rational points $\mathcal{L}(X)(K)$ are exactly formal arcs Spec $K[[t]] \rightarrow X$, for every field $K$ containing $k$. We also introduced in [12] an important boolean subalgebra $\mathbf{B}_{X}$ of the algebra of subsets of $\mathcal{L}(X)$, the subalgebra of semi-algebraic subsets of $\mathcal{L}(X)$. Semi-algebraic subsets of $\mathcal{L}(X)$ are definable in the language of valued fields. We defined a measure $\mu$ on $\mathbf{B}_{X}$ with values - following ideas of Kontsevich - into the ring $\widehat{K}_{0}\left(\mathrm{Sch}_{k}\right)$ which is a certain completion of the localization of the ring $K_{0}\left(\mathrm{Sch}_{k}\right)$ with respect to the class of the affine line. In fact, there exists a general notion of measurable subset of $\mathcal{L}(X)$ for $\mu$, developed in the appendix of [14], and $\mu$ may be extended in a natural way to these measurable subsets. See also [11] and [13] for variants with values into Grothendieck groups of motives. This theory, which maybe should be called geometric motivic integration, is not well suited for the aims of the present paper, the main reason being that at the residue field level, images by morphisms are considered geometrically, in the sense of algebraic geometry, and not at the level of looking to rational points, which corresponds to leaving the world of algebraic geometry (polynomial equations or inequations) for the world of first order logic of fields (formulas with polynomials and quantifiers). We are thus led to develop a different kind of motivic integration theory, arithmetic motivic integration, which takes rationality properties into account. This theory assigns to every definable subset of $\mathcal{L}(X)$ an element in the ring $\widehat{K}_{0}^{v}\left(\operatorname{Mot}_{k, \overline{\mathbf{Q}}}\right) \otimes \mathbf{Q}$, where $\widehat{K}_{0}^{v}\left(\operatorname{Mot}_{k, \overline{\mathbf{Q}}}\right)$ is a certain completion of the localization of $K_{0}^{v}\left(\operatorname{Mot}_{k, \overline{\mathbf{Q}}}\right)$ with respect to the Lefschetz motive. Its very definition relies on our construction of assigning a virtual motive $\chi_{c}(\varphi)$ to a formula $\varphi$ in the first order language of rings with coefficients in $k$, so it involves in an essential way arithmetical tools such as decomposition subgroups, Chebotarev's Theorem and pseudo-finite fields.

0.7. Let us now briefly review the contents of the paper. After a first section devoted to preliminaries, we develop in section 2 what we need on Galois stratifications. In section 3 we give our basic construction of assigning a virtual motive to a formula in the first order language of rings. First order formulas define subsets of the affine space. Since we need to perform some geometric constructions like blowing ups, we introduce a new class of objects, which are more geometric in nature than formulas, which we call "definable subassignments" since they are not in general functors. Section 4 is devoted to definable subassignments for rings and 
section 5 to definable subassignments for power series rings. In section 6 we develop the basic theory of arithmetic motivic integration. Section 7 is devoted to general rationality results, in the spirit of [7] and [12. We are then able in section 8 to prove that arithmetic motivic integration specializes to $p$-adic integration. In section 9 we define the arithmetical Poincaré series $P_{\mathrm{ar}}(T)$ for varieties $X$ over a field $k$ of characteristic zero and we show it specializes to the $p$-adic Poincaré series when $k$ is a number field. The Poincaré series $P_{\mathrm{ar}}(T)$ seems to contain much more interesting geometric information about the variety $X$ than its geometric counterpart $P_{\text {geom }}(T)$ introduced in [12. As an example, we compute both series for branches of plane curves in section 10 In that case the poles of $P_{\mathrm{ar}}(T)$ completely determine the Puiseux pairs of the branch, while $P_{\text {geom }}(T)$ allows one only to recover the multiplicity at the origin.

\section{Contents}

1. Preliminaries

2. Galois stratifications 434

3. Assigning virtual motives to formulas 438

4. Definable subassignments for rings 448

5. Definable subassignments for power series rings 449

6. Arithmetic motivic integration on definable sets 455

7. Rationality results 457

8. Arithmetic motivic integration specializes to $p$-adic integration 460

9. Arithmetic Poincaré series versus geometric Poincaré series 464

10. An example: Branches of plane curves 466

References 468

\section{Preliminaries}

1.1. For any ring $R$, by the first order language of rings with coefficients in $R$ we shall mean the first order language (in the sense of logic), with symbols to denote $+,-, \times, 0,1$, and for each element of $R$ a symbol to denote that element. As for any first order language, formulas are built up from the above symbols together with variables, the logical connectives $\wedge$ (and), $\vee$ (or), $\neg$ (not), the quantifiers $\exists, \forall$ and the equality symbol $=$.

Let $S$ be a scheme. By a variety over $S$ or $S$-variety we shall mean a separated and reduced scheme of finite type over $S$. When $S=\operatorname{Spec} R$ is affine we shall also say variety over $R$ or $R$-variety.

1.2. Let $k$ be a field. We shall denote by $K_{0}\left(\operatorname{Sch}_{k}\right)$ the Grothendieck ring of algebraic varieties over $k$. It is the ring generated by symbols $[S]$, for $S$ an algebraic variety over $k$, with the relations $[S]=\left[S^{\prime}\right]$ if $S$ is isomorphic to $S^{\prime},[S]=\left[S \backslash S^{\prime}\right]+\left[S^{\prime}\right]$ if $S^{\prime}$ is closed in $S$ and $\left[S \times S^{\prime}\right]=[S]\left[S^{\prime}\right]$. Note that, for $S$ an algebraic variety over $k$, the mapping $S^{\prime} \mapsto\left[S^{\prime}\right]$ from the set of closed subvarieties of $S$ extends uniquely to a mapping $W \mapsto[W]$ from the set of constructible subsets of $S$ to $K_{0}\left(\operatorname{Sch}_{k}\right)$, satisfying $\left[W \cup W^{\prime}\right]=[W]+\left[W^{\prime}\right]-\left[W \cap W^{\prime}\right]$. We set $\mathbf{L}:=\left[\mathbf{A}_{k}^{1}\right]$ and $K_{0}\left(\mathrm{Sch}_{k}\right)_{\text {loc }}:=K_{0}\left(\mathrm{Sch}_{k}\right)\left[\mathbf{L}^{-1}\right]$. 
Let $S$ be an algebraic variety over $k$. We write $\operatorname{dim} S \leq n$ if all irreducible components of $S$ have dimension $\leq n$. Similarly, for $M$ in $K_{0}\left(\operatorname{Sch}_{k}\right)$, we write $\operatorname{dim} M \leq n$ if $M$ may be expressed as a linear combination of algebraic varieties with $\operatorname{dim} \leq n$. For $m$ in $\mathbf{Z}$, we denote by $F^{m} K_{0}\left(\operatorname{Sch}_{k}\right)_{\text {loc }}$ the subgroup of $K_{0}\left(\operatorname{Sch}_{k}\right)_{\text {loc }}$ generated by elements of the form $[S] \mathbf{L}^{-i}$ with $i-\operatorname{dim} S \geq m$. This defines a decreasing filtration $F^{m}$ on $K_{0}\left(\operatorname{Sch}_{k}\right)_{\text {loc }}$. We denote by $\widehat{K}_{0}\left(\operatorname{Sch}_{k}\right)$ the completion of $K_{0}\left(\mathrm{Sch}_{k}\right)_{\text {loc }}$ with respect to that filtration. We do not know whether the natural morphism $K_{0}\left(\operatorname{Sch}_{k}\right)_{\text {loc }} \rightarrow \widehat{K}_{0}\left(\operatorname{Sch}_{k}\right)$ is injective or not. We denote by $\bar{K}_{0}\left(\operatorname{Sch}_{k}\right)_{\text {loc }}$ the image of $K_{0}\left(\operatorname{Sch}_{k}\right)_{\text {loc }}$ in $\widehat{K}_{0}\left(\operatorname{Sch}_{k}\right)$.

1.3. We denote by $\operatorname{Mot}_{k}$ the category of Chow motives over $k$, with coefficients in Q. It is a pseudo-abelian category and we denote by $K_{0}\left(\operatorname{Mot}_{k}\right)$ its Grothendieck group. It may also be defined as the abelian group associated to the monoid of isomorphism classes of objects in $\operatorname{Mot}_{k}$ with respect to $\oplus$. Let us recall (see [28] for more details) that objects in $\operatorname{Mot}_{k}$ are just triples $(S, p, n)$ with $S$ proper and smooth over $k, p$ an idempotent correspondence with coefficients in $\mathbf{Q}$ on $S$ and $n$ in $\mathbf{Z}$. The tensor product on $\operatorname{Mot}_{k}$ induces a product on $K_{0}\left(\operatorname{Mot}_{k}\right)$ which provides $K_{0}\left(\operatorname{Mot}_{k}\right)$ with a natural ring structure.

Assume now that $k$ is of characteristic zero. By a result of Gillet and Soulé [20] and Guillén and Navarro Aznar [21] there exists a unique morphism of rings

$$
\chi_{c}: K_{0}\left(\mathrm{Sch}_{k}\right) \longrightarrow K_{0}\left(\operatorname{Mot}_{k}\right)
$$

such that $\chi_{c}([S])=[h(S)]$ for $S$ projective and smooth, where $h(S)$ denotes the Chow motive associated to $S$, i.e. $h(S)=(S$, id, 0$)$. Let us still denote by $\mathbf{L}$ the image of $\mathbf{L}$ by $\chi_{c}$. Since $\mathbf{L}=[(\operatorname{Spec} k, \mathrm{id},-1)]$, it is invertible in $K_{0}\left(\operatorname{Mot}_{k}\right)$, hence $\chi_{c}$ can be extended uniquely to a ring morphism

$$
\chi_{c}: K_{0}\left(\operatorname{Sch}_{k}\right)_{\text {loc }} \longrightarrow K_{0}\left(\operatorname{Mot}_{k}\right) \text {. }
$$

Let us denote by $\operatorname{Mot}_{k, \overline{\mathbf{Q}}}$ the category of Chow motives over $k$, with coefficients in $\overline{\mathbf{Q}}$, and by $K_{0}\left(\operatorname{Mot}_{k, \overline{\mathbf{Q}}}\right)$ its Grothendieck group. Objects in $\operatorname{Mot}_{k, \overline{\mathbf{Q}}}$ are triples $(S, p, n)$ with $S$ proper and smooth over $k, p$ an idempotent correspondence with coefficients in $\overline{\mathbf{Q}}$ on $X$ and $n$ in $\mathbf{Z}$; see, e.g., 11 for more details. We denote by $K_{0}^{v}\left(\operatorname{Mot}_{k, \overline{\mathbf{Q}}}\right)$, resp. $K_{0}^{v}\left(\operatorname{Mot}_{k, \overline{\mathbf{Q}}}\right)_{\text {loc }}$, the image of $K_{0}\left(\operatorname{Sch}_{k}\right)$, resp. $K_{0}\left(\operatorname{Sch}_{k}\right)_{\text {loc }}$, in $K_{0}\left(\operatorname{Mot}_{k, \overline{\mathbf{Q}}}\right)$ by the morphism, which we will still denote by $\chi_{c}$, obtained by composition of $\chi_{c}$ with the natural morphism $K_{0}\left(\operatorname{Mot}_{k}\right) \rightarrow K_{0}\left(\operatorname{Mot}_{k, \overline{\mathbf{Q}}}\right)$. We denote by $F^{m}$ the decreasing filtration on $K_{0}^{v}\left(\operatorname{Mot}_{k, \overline{\mathbf{Q}}}\right)_{\text {loc }}$ which is the image of the filtration $F^{m}$ on $K_{0}\left(\operatorname{Sch}_{k}\right)_{\text {loc }}$, and by $\widehat{K}_{0}^{v}\left(\operatorname{Mot}_{k, \overline{\mathbf{Q}}}\right)$ the completion of $K_{0}^{v}\left(\operatorname{Mot}_{k, \overline{\mathbf{Q}}}\right)_{\text {loc }}$ with respect to the filtration $F^{m}$. We also define $\bar{K}_{0}^{v}\left(\operatorname{Mot}_{k, \overline{\mathbf{Q}}}\right)_{\text {loc }}$ as the image of $K_{0}^{v}\left(\operatorname{Mot}_{k, \overline{\mathbf{Q}}}\right)_{\text {loc }}$ in $\widehat{K}_{0}^{v}\left(\operatorname{Mot}_{k, \overline{\mathbf{Q}}}\right)$. We set

$$
\begin{gathered}
K_{0}^{v}\left(\operatorname{Mot}_{k, \overline{\mathbf{Q}}}\right)_{\mathbf{Q}}:=K_{0}^{v}\left(\operatorname{Mot}_{k, \overline{\mathbf{Q}}}\right) \otimes \mathbf{Q}, K_{0}^{v}\left(\operatorname{Mot}_{k, \overline{\mathbf{Q}}}\right)_{\mathrm{loc}, \mathbf{Q}}:=K_{0}^{v}\left(\operatorname{Mot}_{k, \overline{\mathbf{Q}}}\right)_{\mathrm{loc}} \otimes \mathbf{Q}, \\
\bar{K}_{0}^{v}\left(\operatorname{Mot}_{k, \overline{\mathbf{Q}}}\right)_{\mathrm{loc}, \mathbf{Q}}:=\bar{K}_{0}^{v}\left(\operatorname{Mot}_{k, \overline{\mathbf{Q}}}\right)_{\mathrm{loc}} \otimes \mathbf{Q} \text { and } \widehat{K}_{0}^{v}\left(\operatorname{Mot}_{k, \overline{\mathbf{Q}}}\right)_{\mathbf{Q}}:=\widehat{K}_{0}^{v}\left(\operatorname{Mot}_{k, \overline{\mathbf{Q}}}\right) \otimes \mathbf{Q} .
\end{gathered}
$$

We denote by $F^{m} \widehat{K}_{0}^{v}\left(\operatorname{Mot}_{k, \overline{\mathbf{Q}}}\right)_{\mathbf{Q}}$ the filtration on $\widehat{K}_{0}^{v}\left(\operatorname{Mot}_{k, \overline{\mathbf{Q}}}\right)_{\mathbf{Q}}$ naturally induced by $F^{m}$.

If $k^{\prime}$ is a field containing $k$ and $M$ belongs to $\operatorname{Mot}_{k}$, we shall denote by $M \otimes k^{\prime}$ the same object but considered as an element of $\operatorname{Mot}_{k^{\prime}}$. Similarly, one denotes by $M \otimes k^{\prime}$ the image of an element $M$ in any of the preceding rings relative to $k$ in the similar ring relative to $k^{\prime}$. 


\section{GAlois Stratifications}

2.1. Galois stratifications. Let $A$ be an integral and normal scheme. A morphism of schemes $h: C \rightarrow A$ is a Galois cover if $C$ is integral, $h$ is étale (hence $C$ is normal) and there is a finite group $G=G(C / A)$, the Galois group, acting on $C$ such that $A$ is isomorphic to the quotient $C / G$ in such a way that $h$ is the composition of the quotient morphism with the isomorphism. Isomorphisms of Galois covers are defined in the usual way. If $A^{\prime}$ is a locally closed integral and normal subscheme of $A$, let $C^{\prime}$ denote any connected component of $C \cap h^{-1}\left(A^{\prime}\right)$. One defines the restriction of the Galois cover $h: C \rightarrow A$ to $A^{\prime}$ as the Galois cover $h^{\prime}: C^{\prime} \rightarrow A^{\prime}$, with $h^{\prime}$ the restriction of $h$ to $C^{\prime}$. The Galois group $G\left(C^{\prime} / A^{\prime}\right)$ is the decomposition subgroup of $G(C / A)$ at the generic point of $C^{\prime}$. The choice of another connected component would give an isomorphic Galois cover. We say that the Galois cover $h: C \rightarrow A$ is colored if $G(C / A)$ is equipped with a family Con of subgroups of $G(C / A)$ which is stable by conjugation under elements of $G(C / A)$. The restriction of a colored Galois cover $h: C \rightarrow A$ to $A^{\prime}$, a locally closed integral and normal subscheme of $A$, is defined by the family $\mathrm{Con}^{\prime}$ of subgroups of $G\left(C^{\prime} / A^{\prime}\right)$ which belong to Con.

Let $S$ be an integral normal scheme and let $X_{S}$ be a variety over $S$. A normal stratification of $X_{S}$,

$$
\mathcal{A}=\left\langle X_{S}, C_{i} / A_{i} \mid i \in I\right\rangle
$$

is a partition of $X_{S}$ into a finite set of integral and normal locally closed $S$ subschemes $A_{i}, i \in I$, each equipped with a Galois cover $h_{i}: C_{i} \rightarrow A_{i}$. A normal stratification

$$
\mathcal{A}^{\prime}=\left\langle X_{S}, C_{i}^{\prime} / A_{i}^{\prime} \mid i^{\prime} \in I^{\prime}\right\rangle
$$

will be said to be finer than $\mathcal{A}$ if, for each $i$ in $I^{\prime}, A_{i}^{\prime}$ is contained in some $A_{j}$ and $C_{i}^{\prime} \rightarrow A_{i}^{\prime}$ is isomorphic to the restriction of $C_{j} \rightarrow A_{j}$ to $A_{i}^{\prime}$ as a Galois cover. We will say that normal stratifications $\mathcal{A}$ and $\mathcal{A}^{\prime}$ are isomorphic if there exists a normal stratification $\mathcal{A}^{\prime \prime}$ which is finer than both $\mathcal{A}$ and $\mathcal{A}^{\prime}$.

A normal stratification $\mathcal{A}$ may be augmented to a Galois stratification

$$
\mathcal{A}=\left\langle X_{S}, C_{i} / A_{i}, \operatorname{Con}\left(A_{i}\right) \mid i \in I\right\rangle
$$

if, for each $i \in I, \operatorname{Con}\left(A_{i}\right)$ is a family of subgroups of $G\left(C_{i} / A_{i}\right)$ which is stable by conjugation under elements in $G\left(C_{i} / A_{i}\right)$, i.e. $\left(C_{i} \rightarrow A_{i}, \operatorname{Con}\left(A_{i}\right)\right)$ is a colored Galois cover. One defines similarly as before finer and isomorphic Galois stratifications. In general it will be harmless to identify isomorphic Galois stratifications.

To any $S$-constructible subset $W$ of $X_{S}$ one may associate a well-defined (up to isomorphism) Galois stratification by taking any normal stratification with all strata contained either in $W$ or in its complement, by taking the identity morphism as Galois cover on each stratum, and taking for $\operatorname{Con}\left(A_{i}\right)$ the family consisting of the group with one element if $A_{i} \subset W$ and the empty family otherwise.

We define the $\operatorname{support} \operatorname{Supp}(\mathcal{A})$ of a Galois stratification

$$
\mathcal{A}=\left\langle X_{S}, C_{i} / A_{i}, \operatorname{Con}\left(A_{i}\right) \mid i \in I\right\rangle
$$

as the union of the sets $A_{i}$ with $\operatorname{Con}\left(A_{i}\right)$ nonempty. We define the dimension $\operatorname{dim}(\mathcal{A})$ as the maximum of the dimensions of the sets $A_{i}$ with $\operatorname{Con}\left(A_{i}\right)$ nonempty. 
2.2. Galois formulas. Let $U=\operatorname{Spec} R$ be an affine scheme, which we assume to be integral and normal. For any variety $X_{U}$ over $U$ and any closed point $x$ of $U$, we denote by $\mathbf{F}_{x}$ the residual field of $x$ on $U$ and by $X_{x}$ the fiber of $X_{U}$ at $x$. More generally, for any field $M$ containing $\mathbf{F}_{x}$, we shall denote by $X_{x, M}$ the fiber product of $X_{U}$ and Spec $M$ over $x$.

Let $X_{U}$ be a variety over $U$. Let $\mathcal{A}=\left\langle X_{U}, C_{i} / A_{i}, \operatorname{Con}\left(A_{i}\right) \mid i \in I\right\rangle$ be a Galois stratification of $X_{U}$ and let $x$ be a closed point of $U$. Let $M$ be a field containing $\mathbf{F}_{x}$ and let a be an $M$-rational point of $X_{x}$ belonging to $A_{i, x}$. We denote by $\operatorname{Ar}\left(C_{i} / A_{i}, x, \mathbf{a}\right)$ the conjugacy class of subgroups of $G\left(C_{i} / A_{i}\right)$ consisting of the decomposition subgroups at $\mathbf{a}$. We shall write

$$
\operatorname{Ar}(\mathbf{a}) \subset \operatorname{Con}(\mathcal{A})
$$

for

$$
\operatorname{Ar}\left(C_{i} / A_{i}, x, \mathbf{a}\right) \subset \operatorname{Con}\left(A_{i}\right) .
$$

For $x$ a closed point of $U$ and $M$ a field containing $\mathbf{F}_{x}$, we consider the subset

$$
Z(\mathcal{A}, x, M):=\left\{\mathbf{a} \in X_{U}(M) \mid \operatorname{Ar}(\mathbf{a}) \subset \operatorname{Con}(\mathcal{A})\right\}
$$

of $X_{U}(M)$.

Let $\mathcal{A}=\left\langle\mathbf{A}_{U}^{m+n}, C_{i} / A_{i}, \operatorname{Con}\left(A_{i}\right) \mid i \in I\right\rangle$ be a Galois stratification of $\mathbf{A}_{U}^{m+n}$. Let $Q_{1}, \ldots, Q_{m}$ be quantifiers. We denote by $\vartheta$ or by $\vartheta(\mathbf{Y})$ the formal expression

$$
\left(Q_{1} X_{1}\right) \ldots\left(Q_{m} X_{m}\right)[\operatorname{Ar}(\mathbf{X}, \mathbf{Y}) \subset \operatorname{Con}(\mathcal{A})]
$$

with $\mathbf{X}=\left(X_{1}, \ldots, X_{m}\right)$ and $\mathbf{Y}=\left(Y_{1}, \ldots, Y_{n}\right)$. We call $\vartheta(\mathbf{Y})$ a Galois formula over $R$ in the free variables $\mathbf{Y}$.

Now to a Galois formula $\vartheta$, to a closed point $x$ of $U$ and to a field $M$ containing $\mathbf{F}_{x}$, one associates the subset

$$
Z(\vartheta, x, M):=\left\{\mathbf{b}=\left(b_{1}, \ldots, b_{n}\right) \in M^{n} \mid\left(Q_{1} a_{1}\right) \ldots\left(Q_{m} a_{m}\right)[\operatorname{Ar}(\mathbf{a}, \mathbf{b}) \subset \operatorname{Con}(\mathcal{A})]\right\}
$$

of $M^{n}$, where the quantifiers $Q_{1} a_{1}, \ldots, Q_{m} a_{m}$ run over $M$.

Let $\varphi\left(Y_{1}, \ldots, Y_{n}\right)$ be a formula in the first order language of rings with coefficients in the ring $R$ and free variables $Y_{1}, \ldots, Y_{n}$. For every closed point $x$ in $U$ and every field $M$ containing $\mathbf{F}_{x}$ we denote by $Z(\varphi, x, M)$ the subset of $M^{n}$ defined by the (image over $\mathbf{F}_{x}$ of the) formula $\varphi$. Assume now $\varphi$ is in prenex normal form, i.e. a formula of the form

$$
\left(Q_{1} X_{1}\right) \ldots\left(Q_{m} X_{m}\right)\left[\bigvee_{i=1}^{k} \bigwedge_{j=1}^{l} f_{i, j}(\mathbf{X}, \mathbf{Y})=0 \wedge g_{i, j}(\mathbf{X}, \mathbf{Y}) \neq 0\right]
$$

with $f_{i, j}$ and $g_{i, j}$ in $R[\mathbf{X}, \mathbf{Y}]$. The formula between brackets defines a $U$-constructible subset $A$ of $\mathbf{A}_{U}^{m+n}$ to which one associates a Galois stratification as above. In this way one obtains a Galois formula $\vartheta$ over $R$ such that $Z(\vartheta, x, M)=Z(\varphi, x, M)$ for every closed point $x$ in $U$ and every field $M$ containing $\mathbf{F}_{x}$.

2.3. Quantifier elimination for Galois formulas. We recall that a pseudofinite field $F$ is a perfect infinite field which has exactly one extension of each degree and such that every absolutely irreducible variety over $F$ has a rational point over $F$. J. Ax proved [1] that an infinite field $F$ is pseudo-finite if and only if every sentence 1 in the first order language of rings which is true in all finite fields

\footnotetext{
${ }^{1} \mathrm{~A}$ sentence is a formula without free variables.
} 
is also true in $F$. We recall also that the property of being a pseudo-finite field is stable by ultraproducts 2

There exist several versions of quantifier elimination for Galois formulas [19], [16], [18]. The following seems to be best suited for the present work.

2.3.1. Theorem. Let $k$ be a field. Let $\mathcal{A}$ be a Galois stratification of $\mathbf{A}_{k}^{m+n}$ and let $\vartheta$ be a Galois formula

$$
\left(Q_{1} X_{1}\right) \ldots\left(Q_{m} X_{m}\right)[\operatorname{Ar}(\mathbf{X}, \mathbf{Y}) \subset \operatorname{Con}(\mathcal{A})]
$$

with respect to $\mathcal{A}$. There exists a Galois stratification $\mathcal{B}$ of $\mathbf{A}_{k}^{n}$ such that, for every pseudo-finite field $F$ containing $k$,

$$
Z(\vartheta, \operatorname{Spec} k, F)=Z(\mathcal{B}, \operatorname{Spec} k, F) .
$$

Proof. Since pseudo-finite fields are Frobenius fields in the terminology of [18, the result is a special case of Proposition 25.9 of [18].

2.3.2. Corollary. Let $\varphi\left(Y_{1}, \ldots, Y_{n}\right)$ be a formula in the first order language of rings with coefficients in a field $k$ and free variables $Y_{1}, \ldots, Y_{n}$. There exists a Galois stratification $\mathcal{B}$ of $\mathbf{A}_{k}^{n}$ such that, for every pseudo-finite field $F$ containing $k$,

$$
Z(\varphi, \operatorname{Spec} k, F)=Z(\mathcal{B}, \operatorname{Spec} k, F) .
$$

Proof. Take $\varphi^{\prime}$ to be a formula in prenex normal form, which is logically equivalent to $\varphi$. Since, for every pseudo-finite field $F$ containing $k, Z(\varphi, k, F)=Z\left(\varphi^{\prime}, k, F\right)$, the result follows from Theorem 2.3.1] and the observation made at the end of 2.2 .

2.3.3. Remark. It follows from [18 that there is an effective algorithm to determine a Galois stratification $\mathcal{B}$ in Theorem 2.3.1 and Corollary 2.3.2.

2.4. Let $k$ be a field and let $\varphi$ and $\varphi^{\prime}$ be two formulas in the first order language of rings with coefficients in $k$ and free variables $X_{1}, \ldots, X_{n}$. Let $F$ be a field containing $k$. Recall that one says that $\varphi$ and $\varphi^{\prime}$ are equivalent in $F$ if they define the same subsets in $F^{n}$. We define the equivalence relation $\approx$ by $\varphi \approx \varphi^{\prime}$ if $\varphi$ is equivalent to $\varphi^{\prime}$ in every pseudo-finite field $F$ containing $k$. There is also a weaker equivalence relation $\equiv$ defined as follows. Let $\varphi$ and $\varphi^{\prime}$ be two formulas in the first order language of fields with coefficients in $k$ and free variables $X_{1}, \ldots, X_{n}$ and $X_{1}, \ldots, X_{n^{\prime}}$, respectively. We write $\varphi \equiv \varphi^{\prime}$ if there exists a formula $\psi$ in the first order language of rings with coefficients in $k$ and free variables $X_{1}, \ldots, X_{n+n^{\prime}}$, such that, for every pseudo-finite field $F$ containing $k$, the subset $Z(\psi, \operatorname{Spec} k, F)$ of $F^{n+n^{\prime}}$ is the graph of a bijection between $Z(\varphi, \operatorname{Spec} k, F)$ and $Z\left(\varphi^{\prime}, \operatorname{Spec} k, F\right)$. In a few cases we shall write $\approx_{k}$ and $\equiv_{k}$ to keep track of the field $k$.

To get a more concrete interpretation of the equivalence relations $\approx$ and $\equiv$, we shall assume now that $k$ is a field of characteristic zero which is the field of fractions of a normal domain $R$ of finite type over $\mathbf{Z}$. We set $U=\operatorname{Spec} R$, and, for any nonzero element $f$ of $R$, we set $U_{f}=\operatorname{Spec} R\left[f^{-1}\right]$. Let $\mathcal{A}_{U}$ be a normal (resp. Galois) stratification of $\mathbf{A}_{U}^{n}$. By base change over Spec $k$, one obtains a normal (resp. Galois) stratification of $\mathbf{A}_{k}^{n}$, which we will denote by $\mathcal{A}_{U} \otimes k$. Conversely, any normal or Galois stratification of $\mathbf{A}_{k}^{n}$ may be obtained in this way, at the cost of replacing $U$ by some localization $U_{f}$.

\footnotetext{
${ }^{2}$ See, e.g., 18 for the definition of ultraproducts.
} 
2.4.1. Proposition. (1) Let $\varphi$ and $\varphi^{\prime}$ be two formulas in the first order language of rings with coefficients in $k$ and free variables $X_{1}, \ldots, X_{n}$. Viewing $\varphi$ and $\varphi^{\prime}$ as formulas in the first order language of rings with coefficients in $R_{f}$, for a suitable $f$, we have $\varphi \approx \varphi^{\prime}$ if and only if, for a suitable nonzero multiple $f^{\prime}$ of $f, Z\left(\varphi, x, \mathbf{F}_{x}\right)=Z\left(\varphi^{\prime}, x, \mathbf{F}_{x}\right)$, for every closed point $x$ of $U_{f^{\prime}}$.

(2) Let $\varphi$ and $\varphi^{\prime}$ be two formulas in the first order language of rings with coefficients in $k$ and free variables $X_{1}, \ldots, X_{n}$ and $X_{1}, \ldots, X_{n^{\prime}}$, respectively. Viewing $\varphi$ and $\varphi^{\prime}$ as formulas in the first order language of rings with coefficients in $R_{f}$, for a suitable $f$, we have $\varphi \equiv \varphi^{\prime}$ if and only if, for a suitable nonzero multiple $f^{\prime}$ of $f$, there exists a formula $\psi$ in the first order language of rings with coefficients in $R_{f^{\prime}}$ and free variables $X_{1}, \ldots, X_{n+n^{\prime}}$, such that, for every closed point $x$ of $U_{f^{\prime}}$, the subset $Z\left(\psi, x, \mathbf{F}_{x}\right)$ of $\mathbf{F}_{x}^{n+n^{\prime}}$ is the graph of a bijection between $Z\left(\varphi, x, \mathbf{F}_{x}\right)$ and $Z\left(\varphi^{\prime}, x, \mathbf{F}_{x}\right)$.

Proof. This follows from Proposition 2.4.2 applied to the sentences

$$
\forall x\left(\varphi(x) \longleftrightarrow \varphi^{\prime}(x)\right)
$$

and

$$
\left[\forall x\left(\varphi(x) \rightarrow \exists ! x^{\prime}:\left(\varphi^{\prime}\left(x^{\prime}\right) \wedge \psi\left(x, x^{\prime}\right)\right)\right)\right] \wedge\left[\forall x^{\prime}\left(\varphi^{\prime}\left(x^{\prime}\right) \rightarrow \exists ! x:\left(\varphi(x) \wedge \psi\left(x, x^{\prime}\right)\right)\right)\right],
$$

in cases (1) and (2), respectively.

2.4.2. Proposition. Let $k$ be a field of characteristic zero which is the field of fractions of a normal domain $R$ of finite type over $\mathbf{Z}$. Let $\vartheta$ be a Galois formula over $R$ (as in 2.2) with no free variables. Then $\vartheta$ is true in every pseudo-finite field $F$ containing $k$ if and only if there exists $f$ in $R \backslash\{0\}$, such that, for every closed point $x$ in Spec $R_{f}$, the formula $\vartheta$ is true in $\mathbf{F}_{x}$, i.e. $Z\left(\vartheta, x, \mathbf{F}_{x}\right)=\mathbf{A}_{\mathbf{F}_{x}}^{0}\left(\mathbf{F}_{x}\right)$.

Proof. We first assume that $\vartheta$ is true in every pseudo-finite field $F$ containing $k$. If, for every $f$ in $R \backslash\{0\}$, there exists a closed point $x$ in Spec $R_{f}$ such that $\vartheta$ is false in $\mathbf{F}_{x}$, a suitable ultraproduct of the fields $\mathbf{F}_{x}$ would yield a pseudo-finite field containing $k$ in which $\vartheta$ is false, since the ultraproduct construction commutes in the present case with the Artin symbol.

Conversely, suppose that there exists $f$ in $R \backslash\{0\}$ such that, for every closed point $x$ in Spec $R_{f}$, the formula $\vartheta$ is true in $\mathbf{F}_{x}$. By the quantifier elimination Theorem 2.3.1, there exists a Galois formula $\mathcal{B}$, over a suitable $R_{f}$, with no free variables and no quantifiers, such that $\vartheta \leftrightarrow \mathcal{B}$ holds in every pseudo-finite field $F$ containing $k$. It follows from the first part of the proof that, maybe only after replacing $f$ by a multiple, $\vartheta \leftrightarrow \mathcal{B}$ holds also in $\mathbf{F}_{x}$ for every closed point $x$ in Spec $R_{f}$.

Thus we may suppose that $\vartheta$ has no quantifiers. Let $\mathcal{A}$ be the Galois stratification over $R$ belonging to $\vartheta$. Because $\vartheta$ has no free variables, $\mathcal{A} \otimes k$ consists of only one cover $\operatorname{Spec} L \rightarrow \operatorname{Spec} k$, with $L$ a field which is Galois over $k$.

Assume now that there exists a pseudo-finite field $F$ containing $k$ in which $\vartheta$ is false. Let $\sigma$ be a topological generator of the absolute Galois group of $F$, and denote by $\tau$ the restriction of $\sigma$ to $L$. Then

$$
\operatorname{Ar}(\operatorname{Spec} L / \operatorname{Spec} k, 0, F)=\left\{\alpha\langle\tau\rangle \alpha^{-1} \mid \alpha \in \operatorname{Gal}(L / k)\right\},
$$

where $\langle\tau\rangle$ denotes the subgroup of $\operatorname{Gal}(L / k)$ generated by $\tau$. Since we suppose that $\vartheta$ is false in $F$, we have $\langle\tau\rangle \not \subset \operatorname{Con}(\mathcal{A})$. By Chebotarev's Theorem for $\operatorname{Spec} R$ 
(see, e.g., [29]), there exists a closed point $x$ of $\operatorname{Spec} R_{f}$ such that

$$
\operatorname{Ar}(\operatorname{Spec} L / \operatorname{Spec} k, x, 0)=\left\{\alpha\langle\tau\rangle \alpha^{-1} \mid \alpha \in \operatorname{Gal}(L / k)\right\} .
$$

Thus $\operatorname{Ar}(\operatorname{Spec} L / \operatorname{Spec} k, x, 0) \not \subset \operatorname{Con}(\mathcal{A})$, whence $\vartheta$ is false in $\mathbf{F}_{x}$, which contradicts our assumption that $\vartheta$ is true in $\mathbf{F}_{x}$.

2.4.3. Lemma. Let $\varphi$ and $\varphi^{\prime}$ be two formulas in the first order language of rings with coefficients in a field $k$. If $\varphi \approx_{k} \varphi^{\prime}$ (resp. $\varphi \equiv_{k} \varphi^{\prime}$ ), then there exists a subfield $k_{0}$ of $k$, of finite type over its prime field and such that the coefficients in $\varphi$ and $\varphi^{\prime}$ belong to $k_{0}$, such that $\varphi \approx_{k_{0}} \varphi^{\prime}$ (resp. $\varphi \equiv_{k_{0}} \varphi^{\prime}$ ).

Proof. First consider the case where $\varphi \approx_{k} \varphi^{\prime}$. Let $\left\{k_{i} \mid i \in \Sigma\right\}$ be the set of all subfields of $k$ which are of finite type over the prime field of $k$ and which contain the constants in $\varphi$ and $\varphi^{\prime}$, and assume there exists for each $i$ in $\Sigma$ a pseudo-finite field $F_{i}$ containing $k_{i}$ in which $\varphi$ is not equivalent to $\varphi^{\prime}$. Choose an ultrafilter $D$ on $\Sigma$ containing $S_{i}:=\left\{j \mid k_{i} \subset k_{j}\right\}$, for every $i$ in $\Sigma$. Let $F$ be the ultraproduct of all the fields $F_{i}$ with respect to $D$. Clearly $F$ is a pseudo-finite field in which $\varphi$ is not equivalent to $\varphi^{\prime}$. Moreover $k$ is imbedded in $F$ by the map $a \mapsto\left(a_{i}\right)_{i \in I}$ $\bmod D$, where $a_{i}=a$ if $a \in k_{i}$ and $a_{i}=0$ if $a \notin k_{i}$. This contradicts $\varphi \approx \varphi^{\prime}$. The proof for $\equiv$ is just the same, considering now the set $\left\{k_{i} \mid i \in \Sigma\right\}$ of all subfields of $k$ which are of finite type over the prime field of $k$ and contain the constants in $\varphi, \varphi^{\prime}$ and $\psi$, where $\psi$ is a formula satisfying the conditions in the definition of $\equiv$ and replacing everywhere " $\varphi$ is not equivalent to $\varphi^{\prime}$ in the pseudo-finite field $M$ " by " $Z(\psi, \operatorname{Spec} k, M)$ is not the graph of a bijection between $Z(\varphi, \operatorname{Spec} k, M)$ and $Z\left(\varphi^{\prime}, \operatorname{Spec} k, M\right) "$

The following quantifier elimination statement follows directly from Theorem 2.3 .1 and Proposition 2.4.2.

2.4.4. Proposition. Let $U=\operatorname{Spec} R$ be an affine scheme of finite type over $\mathbf{Z}$, integral, normal and of characteristic zero. Let $\mathcal{A}$ be a Galois stratification of $\mathbf{A}_{U}^{m+n}$ and let $\vartheta$ be a Galois formula

$$
\left(Q_{1} X_{1}\right) \ldots\left(Q_{m} X_{m}\right)[\operatorname{Ar}(\mathbf{X}, \mathbf{Y}) \subset \operatorname{Con}(\mathcal{A})]
$$

with $Q_{1}, \ldots, Q_{m}$ quantifiers. There exist a nonzero element $f$ of $R$ and a Galois stratification $\mathcal{B}$ of $\mathbf{A}_{U_{f}}^{n}$ such that, for every closed point $x$ of $U_{f}$,

$$
Z\left(\vartheta, x, \mathbf{F}_{x}\right)=Z\left(\mathcal{B}, x, \mathbf{F}_{x}\right) .
$$

2.4.5. Corollary. Let $\varphi\left(Y_{1}, \ldots, Y_{n}\right)$ be a formula in the first order language of rings with coefficients in the ring $R$ and free variables $Y_{1}, \ldots, Y_{n}$. There exist a nonzero element $f$ of $R$ and a Galois stratification $\mathcal{B}$ of $\mathbf{A}_{U_{f}}^{n}$ such that, for every closed point $x$ of $U_{f}$,

$$
Z\left(\varphi, x, \mathbf{F}_{x}\right)=Z\left(\mathcal{B}, x, \mathbf{F}_{x}\right) .
$$

\section{Assigning Virtual motives to FORMUlas}

3.1. Motives and group action. Let $k$ be a field of characteristic zero. Let $G$ be a finite group. Let $X$ be an algebraic variety over $k$ endowed with a $G$-action. We say $X$ is a $G$-variety if the $G$-orbit of every closed point in $X$ is contained in an affine open subscheme (this condition is always satisfied when $X$ is quasi-projective). One defines in the usual way isomorphisms and closed immersions of $G$-varieties and so 
one may define a ring $K_{0}\left(\operatorname{Sch}_{k}, G\right)$, the Grothendieck ring of $G$-varieties over $k$, similarly as in 1.2 .

Let $\alpha$ be the character of a representation $G \rightarrow \mathrm{GL}\left(V_{\alpha}\right)$ defined over $\overline{\mathbf{Q}}$. Denote by $n_{\alpha}$ the dimension of $V_{\alpha}$ and consider

$$
p_{\alpha}:=\frac{n_{\alpha}}{|G|} \sum_{g \in G} \alpha^{-1}(g)[g]
$$

the corresponding idempotent in $\overline{\mathbf{Q}}[G]$.

There is a natural ring morphism $\mu$ from $\overline{\mathbf{Q}}[G]$ to the ring of correspondences on $X$ with coefficients in $\overline{\mathbf{Q}}$ sending a group element $g$ onto the graph of multiplication by $g$.

We will need the following equivariant version of the result of Gillet and Soule [20] and Guillén and Navarro Aznar [21]. We denote by $R_{\overline{\mathbf{Q}}}(G)$ the group of characters of virtual representations of $G$ defined over $\overline{\mathbf{Q}}$.

3.1.1. Theorem. Let $G$ be a finite group. For every virtual character $\alpha$ in $R_{\overline{\mathbf{Q}}}(G)$, there exists a unique morphism of rings

$$
\chi_{c}(-, \alpha): K_{0}\left(\operatorname{Sch}_{k}, G\right) \longrightarrow K_{0}\left(\operatorname{Mot}_{k, \overline{\mathbf{Q}}}\right)
$$

such that:

(1) If $X$ is projective and smooth with $G$-action and $\alpha$ is the character of an irreducible representation defined over $\overline{\mathbf{Q}}, n_{\alpha} \chi_{c}([X], \alpha)$ is equal to the class of the motive $\left(X, \mu\left(p_{\alpha}\right), 0\right)$ in $K_{0}\left(\operatorname{Mot}_{k, \overline{\mathbf{Q}}}\right)$.

(2) For every $G$-variety $X$,

$$
\chi_{c}(X)=\sum_{\alpha} n_{\alpha} \chi_{c}(X, \alpha)
$$

where $\alpha$ runs over all irreducible characters of $G$.

(3) For every $G$-variety $X$, the function $\alpha \mapsto \chi_{c}(X, \alpha)$ is a group morphism $R_{\overline{\mathbf{Q}}}(G) \rightarrow K_{0}\left(\operatorname{Mot}_{k, \overline{\mathbf{Q}}}\right)$.

Proof. This is Theorem 6.1 of [2]. When $G$ is abelian it is Theorem 1.3.1 of [1] where the hypothesis $G$ abelian was not used seriously in the proof.

We shall also use the following result.

3.1.2. Proposition. Let $G$ be a finite group, let $H$ be a subgroup of $G$ and let $X$ be a $G$-variety.

(1) Assume $H$ is a normal subgroup of $G$. Then, for every character $\alpha$ of $G / H$,

$$
\chi_{c}(X / H, \alpha)=\chi_{c}(X, \alpha \circ \varrho)
$$

where $\varrho$ is the projection $G \rightarrow G / H$.

(2) Let $\alpha$ be a character of $H$. Then

$$
\chi_{c}\left(X, \operatorname{Ind}_{H}^{G} \alpha\right)=\chi_{c}(X, \alpha),
$$

viewing $X$ as an $H$-variety in the second term of the equality.

(3) Assume $X$ is isomorphic as a $G$-variety to $\bigoplus_{s \in G / H} s Y$, with $Y$ an $H$-variety. Then, for every character $\alpha$ of $G$,

$$
\chi_{c}(X, \alpha)=\chi_{c}\left(Y, \operatorname{Res}_{H}^{G} \alpha\right) .
$$


Proof. Statements (1) and (3) follow from Proposition 6.3 of [2] applied to the morphism $\varrho: G \rightarrow G / H$ and $H \rightarrow G$, respectively. Similarly, statement (2) follows from a dual form of Proposition 6.3 of [2] which is as follows (notation being as in loc. cit.): for $\psi: G \rightarrow G^{\prime}$ a morphism of finite groups, $\alpha$ a character of $G$ and $X$ a $G^{\prime}$-variety, we have $\chi_{c}\left(\operatorname{Res}_{\psi} X, \alpha\right)=\chi_{c}\left(X, \operatorname{Ind}_{\psi} \alpha\right)$. The proof is just similar to the proof of Proposition 6.3 of 2 using the projection formula Corollary 4.3 of [2] and the fact that the functor $h_{c}$ of loc. cit. commutes with $\operatorname{Res}_{\psi}$. Another way to prove (2) and (3) would be to remark that when $X$ is projective and smooth it is just a consequence of Theorem 3.1.1 (1) together with elementary theory of representations of finite groups and then to deduce the result for arbitrary $X$ by additivity of $\chi_{c}$.

Let us denote by $C(G, \overline{\mathbf{Q}})$ the $\overline{\mathbf{Q}}$-vector space of $\mathbf{\mathbf { Q }}$-valued central functions on $G$. For every $W$ in $K_{0}\left(\operatorname{Sch}_{k}, G\right)$, one defines by linearity a morphism of vector spaces $\alpha \mapsto \chi_{c}(W, \alpha)$ from $C(G, \overline{\mathbf{Q}})$ to $K_{0}\left(\operatorname{Mot}_{k, \overline{\mathbf{Q}}}\right) \otimes \overline{\mathbf{Q}}$, expressing a central function as a linear combination of irreducible characters. Now let us denote by $C(G, \mathbf{Q})$ the $\mathbf{Q}$-vector space of $\mathbf{Q}$-central functions $G \rightarrow \mathbf{Q}$, i.e. $\mathbf{Q}$-linear combinations of characters of $\mathbf{Q}$-irreducible representations of $G$ defined over $\mathbf{Q}$. We recall that a central function $\alpha: G \rightarrow \mathbf{Q}$ belongs to $C(G, \mathbf{Q})$ if and only if $\alpha(x)=\alpha\left(x^{\prime}\right)$ for each $x, x^{\prime}$ in $G$ such that $\langle x\rangle$ is conjugate to $\left\langle x^{\prime}\right\rangle$. Here $\langle x\rangle$ denotes the subgroup generated by $x$.

3.1.3. Proposition. Let $G$ be a finite group. For every central function $\alpha: G \rightarrow \mathbf{Q}$ in $C(G, \mathbf{Q})$ and every $W$ in $K_{0}\left(\operatorname{Sch}_{k}, G\right)$, the virtual motive $\chi_{c}(W, \alpha)$ belongs to the image of the morphism of $\mathbf{Q}$-vector spaces

$$
K_{0}\left(\operatorname{Sch}_{k}\right) \otimes \mathbf{Q} \longrightarrow K_{0}\left(\operatorname{Mot}_{k, \overline{\mathbf{Q}}}\right) \otimes \mathbf{Q}
$$

induced by $\chi_{c}$, i.e. is an element of $K_{0}^{v}\left(\operatorname{Mot}_{k, \overline{\mathbf{Q}}}\right)_{\mathbf{Q}}$.

Proof. By a classical result of Emil Artin, $\alpha$ is a Q-linear combination of characters of the form $\operatorname{Ind}_{H}^{G} 1_{H}$ with $H$ a cyclic subgroup of $G$. It follows from Proposition 3.1 .2 that, for every $G$-variety $X$,

$$
\chi_{c}\left(X, \operatorname{Ind}_{H}^{G} 1_{H}\right)=\chi_{c}\left(X, 1_{H}\right)=\chi_{c}(X / H),
$$

where in the middle term $X$ is viewed as an $H$-variety, whence $\chi_{c}\left(X, \operatorname{Ind}_{H}^{G} 1_{H}\right)$ belongs to $K_{0}^{v}\left(\operatorname{Mot}_{k, \overline{\mathbf{Q}}}\right)$.

3.2. Assigning virtual motives to Galois stratifications. Let $k$ be a field of characteristic zero and let $A$ be a normal integral variety over $k$. Let $h: C \rightarrow A$ be a Galois cover with Galois group $G$ and let Con be a family of subgroups of $G$ which is stable by conjugation under elements in $G$. One may associate to these data an element $\chi_{c}(C / A$, Con $)$ of $K_{0}^{v}\left(\operatorname{Mot}_{k, \overline{\mathbf{Q}}}\right)_{\mathbf{Q}}$ as follows. Consider the central function $\alpha_{\text {Con }}$ on $G$ defined by

$$
\alpha_{\text {Con }}(x)= \begin{cases}1 & \text { if }\langle x\rangle \in \text { Con } \\ 0 & \text { if }\langle x\rangle \notin \text { Con. }\end{cases}
$$

Clearly $\alpha_{\text {Con }}$ belongs to $C(G, \mathbf{Q})$, hence, by Proposition 3.1.3, $\chi_{c}\left(C, \alpha_{\text {Con }}\right)$ belongs to $K_{0}^{v}\left(\operatorname{Mot}_{k, \overline{\mathbf{Q}}}\right)_{\mathbf{Q}}$. We set $\chi_{c}(C / A, \operatorname{Con}):=\chi_{c}\left(C, \alpha_{\text {Con }}\right)$. 
Now let $X$ be a variety over $k$ and let $\mathcal{A}=\left\langle X, C_{i} / A_{i}, \operatorname{Con}\left(A_{i}\right) \mid i \in I\right\rangle$ be a Galois stratification of $X$. We define the element

$$
\chi_{c}(\mathcal{A}):=\sum_{i \in I} \chi_{c}\left(C_{i} / A_{i}, \operatorname{Con}\left(A_{i}\right)\right)
$$

in $K_{0}^{v}\left(\operatorname{Mot}_{k, \overline{\mathbf{Q}}}\right)_{\mathbf{Q}}$.

3.2.1. Proposition. Let $X$ be a variety over $k$ and let $\mathcal{A}$ be a Galois stratification of $X$. The element $\chi_{c}(\mathcal{A})$ in $K_{0}^{v}\left(\operatorname{Mot}_{k, \overline{\mathbf{Q}}}\right)_{\mathbf{Q}}$ depends only on the isomorphism class of the Galois stratification $\mathcal{A}$ of $X$.

Proof. The proof follows directly from the additivity of $\chi_{c}$ and Proposition 3.1 .2 (3).

3.2.2. Proposition. Let $k$ be a field of characteristic zero which is the field of fractions of a normal domain $R$ of finite type over $\mathbf{Z}$. Let $A$ be a normal integral variety over $U:=\operatorname{Spec} R$ and consider a Galois stratification $\mathcal{A}=\langle A, C / A, \operatorname{Con}(A)\rangle$ consisting of a single colored Galois cover. Let $C^{\prime} \rightarrow C$ be a Galois cover such that the induced map $C^{\prime} \rightarrow A$ is a Galois cover. Consider the family $\operatorname{Con}^{\prime}(A)$ of subgroups of $G\left(C^{\prime} / A\right)$ which are mapped onto subgroups in $\operatorname{Con}(A)$ by the projection $\varrho: G\left(C^{\prime} / A\right) \rightarrow G(C / A)$ and denote by $\mathcal{A}^{\prime}$ the Galois stratification $\mathcal{A}^{\prime}=$ $\left\langle A, C^{\prime} / A, \operatorname{Con}^{\prime}(A)\right\rangle$.

(1) For every closed point $x$ of $U$ and every field $M$ containing $\mathbf{F}_{x}, Z(\mathcal{A}, x, M)=$ $Z\left(\mathcal{A}^{\prime}, x, M\right)$.

(2) We have

$$
\chi_{c}(\mathcal{A} \otimes k)=\chi_{c}\left(\mathcal{A}^{\prime} \otimes k\right) .
$$

Proof. The first statement is clear and the second follows from Proposition 3.1 .2 (1), since $\alpha_{\operatorname{Con}^{\prime}(A)}=\alpha_{\operatorname{Con}(A)} \circ \varrho$.

3.3. Frobenius action. We assume in this subsection that $k$ is a field of characteristic zero which is the field of fractions of a normal domain $R$ of finite type over Z. We again set $U=\operatorname{Spec} R$.

Let us fix a prime number $\ell$ and denote by $G_{k}$ the absolute Galois group of $k$. We denote by $K_{0}\left(\overline{\mathbf{Q}}_{\ell}, G_{k}\right)$ the Grothendieck group of the abelian category of finite dimensional $\overline{\mathbf{Q}}_{\ell}$-vector spaces with continuous $G_{k}$-action. For every closed point $x$ of $U$, we denote by Frob ${ }_{x}$ the geometric Frobenius automorphism over the field $\mathbf{F}_{x}$. Taking the trace of Frob $x$ on the invariants by inertia gives rise to a ring morphism

$$
K_{0}\left(\overline{\mathbf{Q}}_{\ell}, G_{k}\right) \otimes \mathbf{Q} \longrightarrow \overline{\mathbf{Q}}_{\ell} .
$$

By composition with the morphism

$$
\mathrm{É}_{\ell}: K_{0}^{v}\left(\operatorname{Mot}_{k, \overline{\mathbf{Q}}}\right) \otimes \mathbf{Q} \longrightarrow K_{0}\left(\overline{\mathbf{Q}}_{\ell}, G_{k}\right) \otimes \mathbf{Q}
$$

induced by étale $\ell$-adic realization, one defines a ring morphism

$$
\operatorname{Tr} \operatorname{Frob}_{x}: K_{0}^{v}\left(\operatorname{Mot}_{k, \overline{\mathbf{Q}}}\right) \otimes \mathbf{Q} \longrightarrow \overline{\mathbf{Q}}_{\ell}
$$

3.3.1. Proposition. Let $X_{U}$ be a variety over $U$ and let $\mathcal{A}$ be a Galois stratification of $X_{U}$. There exists a nonzero element $f$ in $R$ such that, for every closed point $x$ of $U_{f}$,

$$
\operatorname{Tr} \operatorname{Frob}_{x}\left(\chi_{c}(\mathcal{A} \otimes k)\right)=\operatorname{card} Z\left(\mathcal{A}, x, \mathbf{F}_{x}\right) .
$$

Proof. The proof follows directly from the next lemma. 
3.3.2. Lemma. Let $A$ be a normal variety over $U$, let $h: C \rightarrow A$ be a Galois cover with Galois group $G$ and let Con be a family of subgroups of $G$ which is stable by conjugation under elements in $G$. There exists a nonzero element $f$ in $R$ such that, for every closed point $x$ of $U_{f}$,

$$
\operatorname{Tr}_{\operatorname{Frob}}\left(\chi_{c}(C / A \otimes k, \operatorname{Con})\right)=\operatorname{card}\left\{a \in A\left(\mathbf{F}_{x}\right) \mid \operatorname{Ar}(C / A, x, a) \subset \operatorname{Con}\right\} .
$$

Proof. By its very definition, $\chi_{c}(C / A \otimes k$, Con $)$ is equal to $\chi_{c}\left(C \otimes k, \alpha_{\text {Con }}\right)$. As in the proof of Proposition 3.1.3 we may write $\alpha_{\mathrm{Con}}$ as a $\mathbf{Q}$-linear combination

$$
\alpha_{\text {Con }}=\sum_{H} n_{H} \operatorname{Ind}_{H}^{G} 1_{H}
$$

with $n_{H}$ in $\mathbf{Q}$ and $H$ running over the set of cyclic subgroups of $G$, and it follows from Proposition 3.1.2 that the left hand side of (3.3.1) is equal to

$$
\sum_{H} n_{H} \sum_{i}(-1)^{i} \operatorname{Tr} \operatorname{Frob}_{x}\left(H_{c}^{i}\left(C / H, \overline{\mathbf{Q}}_{\ell}\right)\right) .
$$

The right hand side of (3.3.1) is equal to

$$
\sum_{a \in A\left(\mathbf{F}_{x}\right)} \alpha_{\operatorname{Con}}\left(\operatorname{Frob}_{a}\right)
$$

with Frob $a$ the Frobenius automorphism at $a$ (up to conjugation) and hence may be rewritten as

$$
\sum_{H} n_{H} \operatorname{card}\left\{a \in C / H\left(\mathbf{F}_{x}\right)\right\} .
$$

Now it follows from Grothendieck's trace formula together with standard constructibility and base change theorems for $\ell$-adic cohomology that there exists a nonzero element $f$ in $R$ such that, for every closed point $x$ of $U_{f}$, (3.3.2) is equal to (3.3.3).

In fact, the sets $Z\left(\mathcal{A}, x, \mathbf{F}_{x}\right)$ completely determine the virtual motive $\chi_{c}(\mathcal{A} \otimes k)$ of a Galois stratification over $U$. More precisely:

3.3.3. Theorem. Let $U=\operatorname{Spec} R$ be an affine scheme of finite type over $\mathbf{Z}$, integral, normal and of characteristic zero. Let $X_{U}$ be a variety over $U$ and let $\mathcal{A}$ and $\mathcal{A}^{\prime}$ be Galois stratifications of $X_{U}$. Let $f$ be a nonzero element of $R$. Assume that, for every closed point $x$ of $U_{f}$, the equality $Z\left(\mathcal{A}, x, \mathbf{F}_{x}\right)=Z\left(\mathcal{A}^{\prime}, x, \mathbf{F}_{x}\right)$ holds. Then

$$
\chi_{c}(\mathcal{A} \otimes k)=\chi_{c}\left(\mathcal{A}^{\prime} \otimes k\right) .
$$

Proof. After refining $\mathcal{A}$ and $\mathcal{A}^{\prime}$ one may assume $I=I^{\prime}$ and $A_{i}=A_{i}^{\prime}$ for $i \in I$. Hence it is sufficient to prove the following: Let $A$ be an integral and normal $U$-scheme of finite type and let $h: C \rightarrow A$ and $h^{\prime}: C^{\prime} \rightarrow A$ be Galois covers with Galois groups $G$ and $G^{\prime}$, respectively, provided with a family of subgroups Con (resp. Con') of $G$ (resp. $G^{\prime}$ ) which is stable by conjugation under elements in $G$ (resp. $G^{\prime}$ ). Assume there exists a nonzero element $f$ in $R$ such that for every closed point $x$ in $U_{f}$ and every closed point $y$ of $A_{x}, \operatorname{Ar}(C / A, x, y) \subset$ Con if and only $\operatorname{Ar}\left(C^{\prime} / A, x, y\right) \subset$ Con' $^{\prime}$. Then

$$
\chi_{c}\left(C \otimes k, \alpha_{\text {Con }}\right)=\chi_{c}\left(C^{\prime} \otimes k, \alpha_{\mathrm{Con}^{\prime}}\right) .
$$

But this follows from the more general Lemma 3.3 .4 
3.3.4. Lemma. Let $A$ be an integral and normal $U$-scheme of finite type and let $h: C \rightarrow A$ and $h^{\prime}: C^{\prime} \rightarrow A$ be Galois covers with Galois groups $G$ and $G^{\prime}$, respectively. Take $\alpha \in \mathcal{C}(G, \mathbf{Q})$ and $\alpha^{\prime} \in \mathcal{C}\left(G^{\prime}, \mathbf{Q}\right)$. Assume there exists a nonzero $f$ in $R$ such that for every closed point $x$ in $U_{f}$ and every closed point $y$ of $A_{x}$,

$$
\alpha\left(\operatorname{Frob}_{y}\right)=\alpha^{\prime}\left(\operatorname{Frob}_{y}\right) .
$$

Then

$$
\chi_{c}(C \otimes k, \alpha)=\chi_{c}\left(C^{\prime} \otimes k, \alpha^{\prime}\right) .
$$

Proof. Replacing $\alpha$ and $\alpha^{\prime}$ by multiples, we may assume that $\alpha$ and $\alpha^{\prime}$ are $\mathbf{Z}$-linear combinations of irreducible characters of $G$ and $G^{\prime}$, respectively. By Proposition 3.2.2 maybe after replacing $U$ by $U_{f}$ for some nonzero $f$ in $R$, we can assume $C=C^{\prime}$ and $G=G^{\prime}$ by going to a suitable common Galois cover of $C$ and $C^{\prime}$. By Chebotarev's Theorem, we then have $\alpha=\alpha^{\prime}$, and the result follows.

More generally, the following result holds.

3.3.5. Theorem. Let $U=\operatorname{Spec} R$ be an affine scheme of finite type over $\mathbf{Z}$, integral, normal and of characteristic zero. Let $A$ and $B$ be varieties over $U$ and let $\mathcal{A}$ and $\mathcal{B}$ be Galois stratifications of $A$ and $B$, respectively. Assume there exists a Galois stratification $\mathcal{Z}$ of $A \times B$ and a nonzero element $f$ of $R$ such that, for every closed point $x$ of $U_{f}, Z\left(\mathcal{Z}, x, \mathbf{F}_{x}\right)$ is the graph of a bijection between $Z\left(\mathcal{A}, x, \mathbf{F}_{x}\right)$ and $Z\left(\mathcal{B}, x, \mathbf{F}_{x}\right)$. Then

$$
\chi_{c}(\mathcal{A} \otimes k)=\chi_{c}(\mathcal{B} \otimes k) .
$$

Proof. It is enough to prove that $\chi_{c}(\mathcal{A} \otimes k)=\chi_{c}(\mathcal{Z} \otimes k)$. Hence, by additivity of $\chi_{c}$, one may assume that the Galois stratification $\mathcal{A}$ consists of a single colored Galois cover $(C \rightarrow A, \operatorname{Con}(A))$, with $A$ a normal variety and $\operatorname{Con}(A)$ nonempty. Denote by $Z$ the support of $\mathcal{Z}$ and by $p$ the restriction of the projection $A \times B \rightarrow A$ to $Z$. Write the Galois stratification $\mathcal{Z}$ as

$$
\mathcal{Z}=\left\langle A \times B, W_{i} / Z_{i}, \operatorname{Con}\left(Z_{i}\right) \mid i \in I\right\rangle .
$$

It follows from Chebotarev's Theorem and the Lang-Weil estimate [23] that the morphism $p: Z \rightarrow A$ is generically finite and that $\overline{p(Z)}=A$. Hence, maybe after performing a finite partition of $A$ into locally closed normal subschemes, we may assume that, for every $i$ in $I, Z_{i}$ is mapped by $p$ onto $A$ and that the restriction of $p$ to $Z_{i}$ is a finite étale morphism. Furthermore, maybe after replacing $U$ by $U_{f}$ for a suitable nonzero $f$, we may, by Proposition 3.2.2 replace the $W_{i}$ 's by suitable Galois covers. It follows that, maybe after performing another finite partition of $A$ into locally closed normal subschemes, we may assume that the morphisms $W_{i} \rightarrow A$ obtained by composition with $p$ are all Galois covers and that they are all equal to the same Galois cover $W \rightarrow A$. Moreover we may assume that the cover $W \rightarrow A$ coincides with the cover $C \rightarrow A$. By assumption there exists a nonzero element $f$ of $R$ such that, for every closed point $x$ of $U_{f}$, the map $p$ induces a bijection between $Z\left(\mathcal{Z}, x, \mathbf{F}_{x}\right)$ and $Z\left(\mathcal{A}, x, \mathbf{F}_{x}\right)$. Hence, for such an $f$ and such an $x$, a point $a$ belongs to $Z\left(\mathcal{A}, x, \mathbf{F}_{x}\right)$ if and only if there exists $i$ in $I$ and $\sigma$ in $G(W / A)$ such that $\sigma\left\langle\right.$ Frob $\left._{a}\right\rangle \sigma^{-1}$ belongs to $\operatorname{Con}\left(Z_{i}\right)$. Furthermore, since a given point $a$ in $Z\left(\mathcal{A}, x, \mathbf{F}_{x}\right)$ should be the image under $p$ of a unique point in $Z\left(\mathcal{Z}, x, \mathbf{F}_{x}\right)$, one deduces from Chebotarev's Theorem that, for $a$ in a subset of closed points of $A$ of Dirichlet 
density 1 , the element $i$ in $I$ and the class of $\sigma$ in $G(W / A) / G\left(W / Z_{i}\right)$ should both be unique. Since, for such an $a$,

$$
\alpha_{\operatorname{Con}(A)}\left(\operatorname{Frob}_{a}\right)=\sum_{i \in I} \operatorname{Ind}_{G\left(W / Z_{i}\right)}^{G(W / A)} \alpha_{\operatorname{Con}\left(Z_{i}\right)}\left(\operatorname{Frob}_{a}\right),
$$

it follows, again by Chebotarev's Theorem, that

$$
\alpha_{\operatorname{Con}(A)}=\sum_{i \in I} \operatorname{Ind}_{G\left(W / Z_{i}\right)}^{G(W / A)} \alpha_{\operatorname{Con}\left(Z_{i}\right)} .
$$

By Proposition $3.1 .2(2)$, one deduces now that $\chi_{c}(\mathcal{A} \otimes k)=\chi_{c}(\mathcal{Z} \otimes k)$.

3.4. Assigning virtual motives to formulas. We assume first that $k$ is a field of characteristic zero which is of finite type over $\mathbf{Q}$. We now associate a canonical element $\chi_{c}(\varphi)$ in $K_{0}^{v}\left(\operatorname{Mot}_{k, \overline{\mathbf{Q}}}\right)_{\mathbf{Q}}$ to every formula $\varphi$ in the first order language of rings with coefficients in $k$ and free variables $X_{1}, \ldots, X_{n}$. The construction is as follows. One may view $\varphi$ as a formula in the first order language of rings with coefficients in $R$, with $R$ a normal subring of $k$ which is of finite type over $\mathbf{Z}$, and whose fraction field is $k$. We again set $U=\operatorname{Spec} R$. By Corollary 2.4.5 one may associate to $\varphi$ a Galois stratification $\mathcal{A}$ of $\mathbf{A}_{U_{f}}^{n}$, for a suitable nonzero $f$, such that, for every closed point $x$ of $U_{f}$,

$$
Z\left(\varphi, x, \mathbf{F}_{x}\right)=Z\left(\mathcal{A}, x, \mathbf{F}_{x}\right) .
$$

Clearly, the Galois stratification $\mathcal{A} \otimes k$ is not canonically associated to $\varphi$. Nevertheless, it follows directly from Theorem 3.3 .3 that the virtual motive $\chi_{c}(\mathcal{A} \otimes k)$ in $K_{0}^{v}\left(\operatorname{Mot}_{k, \overline{\mathbf{Q}}}\right)_{\mathbf{Q}}$ is canonically attached to $\varphi$. We shall denote it by $\chi_{c}(\varphi)$.

Now we investigate the behaviour of $\chi_{c}$ under field extensions.

Let $k$ be a field of characteristic zero and let $A$ be a normal integral variety over $k$. Let $h: C \rightarrow A$ be a Galois cover with Galois group $G$. If $k^{\prime}$ is a field containing $k$, the restriction of the morphism $h \otimes k^{\prime}: C \otimes k^{\prime} \rightarrow A \otimes k^{\prime}$ obtained by extension of scalars to a connected component $C^{\prime}$ of $C \otimes k^{\prime}$ defines a Galois cover of $A \otimes k^{\prime}$ with Galois group $G^{\prime}$, the decomposition subgroup of $G$ at the generic point of $C^{\prime}$, whose isomorphism class is independent of the choice of $C^{\prime}$. If the Galois cover is colored by a family Con of subgroups of $G$ which is stable by conjugation under elements in $G$, one considers the family $\mathrm{Con}^{\prime}$ obtained by intersection with $G^{\prime}$. In this way, for $\mathcal{A}$ a Galois stratification of a $k$-variety $X$, one defines a Galois stratification $\mathcal{A} \otimes k^{\prime}$ of $X \otimes k^{\prime}$.

3.4.1. Lemma. Let $k$ be a field of characteristic zero and let $\mathcal{A}$ be a Galois stratification of a k-variety $X$. Then

$$
\chi_{c}\left(\mathcal{A} \otimes k^{\prime}\right)=\chi_{c}(\mathcal{A}) \otimes k^{\prime} .
$$

Proof. It is enough to prove the following: Let $h: C \rightarrow A$ be a Galois cover of $k$-varieties with Galois group $G, C^{\prime}$ a connected component of $C \otimes k^{\prime}, G^{\prime}$ the decomposition subgroup of $G$ at the generic point of $C^{\prime}$, and $\alpha$ a character of $G$. Then $\chi_{c}(C, \alpha) \otimes k^{\prime}=\chi_{c}\left(C^{\prime}, \operatorname{Res}_{G^{\prime}}^{G} \alpha\right)$. Since $\chi_{c}(C, \alpha) \otimes k^{\prime}=\chi_{c}\left(C \otimes k^{\prime}, \alpha\right)$, this is equivalent to the equality $\chi_{c}\left(C \otimes k^{\prime}, \alpha\right)=\chi_{c}\left(C^{\prime}, \operatorname{Res}_{G^{\prime}}^{G} \alpha\right)$ which follows from Proposition $3.1 .2(3)$. 
3.4.2. Lemma. Let $k \subset k^{\prime}$ be fields of finite type over $\mathbf{Q}$. Let $\varphi$ be a formula in the first order language of rings with coefficients in $k$ and free variables $X_{1}, \ldots, X_{n}$. We denote by $\varphi \otimes k^{\prime}$ the same formula considered as a formula with coefficients in $k^{\prime}$. Then

$$
\chi_{c}\left(\varphi \otimes k^{\prime}\right)=\chi_{c}(\varphi) \otimes k^{\prime}
$$

Proof. Using the preceding notations, take a Galois stratification $\mathcal{A}$ of $\mathbf{A}_{U_{f}}^{n}$, such that, for every closed point $x$ of $U_{f}, Z\left(\varphi, x, \mathbf{F}_{x}\right)=Z\left(\mathcal{A}, x, \mathbf{F}_{x}\right)$. By Proposition 2.4.2, $Z(\varphi$, Spec $k, F)=Z(\mathcal{A}$, Spec $k, F)$ for every pseudo-finite field $F$ containing $k$. Hence, $Z\left(\varphi \otimes k^{\prime}\right.$, Spec $\left.k^{\prime}, F\right)=Z\left(\mathcal{A} \otimes k^{\prime}\right.$, Spec $\left.k^{\prime}, F\right)$ for every pseudo-finite field $F$ containing $k^{\prime}$. Let $U^{\prime}=\operatorname{Spec} R^{\prime}$ with $R^{\prime}$ the normalisation of $R$ in $k^{\prime}$. Again by Proposition 2.4.2 we get that for some nonzero element $f^{\prime}$ of $R^{\prime}, Z\left(\varphi \otimes k^{\prime}, x, \mathbf{F}_{x}\right)=$ $Z\left(\mathcal{A} \otimes U_{f^{\prime}}^{\prime}, x, \mathbf{F}_{x}\right)$, for every closed point $x$ of $U_{f^{\prime}}^{\prime}$, with $\mathcal{A} \otimes U_{f^{\prime}}^{\prime}$ the stratification obtained by base change. Hence, by Theorem 3.3.3, $\chi_{c}\left(\varphi \otimes k^{\prime}\right)=\chi_{c}\left(\mathcal{A} \otimes k^{\prime}\right)$ and the result follows by Lemma 3.4.1

Let $k$ be any field of characteristic zero. Let $\varphi$ be a formula in the first order language of rings with coefficients in $k$. We may now associate to $\varphi$ a canonical element $\chi_{c}(\varphi)$ in $K_{0}^{v}\left(\operatorname{Mot}_{k, \overline{\mathbf{Q}}}\right)_{\mathbf{Q}}$ as follows. Take a subfield $k_{0}$ of $k$ which is of finite type over $\mathbf{Q}$ such that $\varphi$ may be viewed as a formula $\varphi_{0}$ in the first order language of rings with coefficients in $k_{0}$. By Lemma 3.4.2 $\chi_{c}\left(\varphi_{0}\right) \otimes k$ does not depend on the choice of $k_{0}$, so we may set $\chi_{c}(\varphi):=\chi_{c}\left(\varphi_{0}\right) \otimes k$.

3.4.3. Proposition. Let $k$ be a field of characteristic zero and let $\varphi$ and $\varphi^{\prime}$ be two formulas in the first order language of rings with coefficients in $k$. If $\varphi \equiv \varphi^{\prime}$, then

$$
\chi_{c}(\varphi)=\chi_{c}\left(\varphi^{\prime}\right)
$$

Proof. By Lemma 2.4.3 we may assume that $k$ is of finite type over $\mathbf{Q}$, so we may apply Proposition 2.4.1 (2) to get a certain formula $\psi$ and then, by Corollary 2.4.5 we may replace the formula $\psi$ by a Galois stratification and the result follows from Theorem 3.3.5.

We shall denote by $\operatorname{Form}_{k}$ the set of formulas in the first order language of rings with coefficients in $k$. If $\varphi$ is a formula in $\operatorname{Form}_{k}$ with $n$ free variables and $\varphi^{\prime}$ is a formula in Form $_{k}$ with $n^{\prime}$ free variables, we shall denote by $\varphi \times \varphi^{\prime}$ the formula with $n+n^{\prime}$ free variables obtained by giving different names to the free variables occurring in $\varphi$ and $\varphi^{\prime}$ and taking the conjunction of $\varphi$ and $\varphi^{\prime}$.

We now list a few properties of $\varphi \mapsto \chi_{c}(\varphi)$.

3.4.4. Proposition. The function $\chi_{c}: \operatorname{Form}_{k} \rightarrow K_{0}^{v}\left(\operatorname{Mot}_{k, \overline{\mathbf{Q}}}\right)_{\mathbf{Q}}$ satisfies the following properties.

(1) Let $\varphi$ and $\varphi^{\prime}$ be formulas with free variables $\left(X_{1}, \ldots, X_{n}\right)$. Then

$$
\chi_{c}\left(\varphi \vee \varphi^{\prime}\right)=\chi_{c}(\varphi)+\chi_{c}\left(\varphi^{\prime}\right)-\chi_{c}\left(\varphi \wedge \varphi^{\prime}\right)
$$

(2) Let $\varphi$ and $\varphi^{\prime}$ be formulas with free variables $\left(X_{1}, \ldots, X_{n}\right)$ and $\left(X_{1}, \ldots, X_{n^{\prime}}\right)$, respectively. Then

$$
\chi_{c}\left(\varphi \times \varphi^{\prime}\right)=\chi_{c}(\varphi) \chi_{c}\left(\varphi^{\prime}\right) .
$$

(3) If $\varphi$ has $n$ free variables, then

(4) $\chi_{c}(0=1)=0$.

$$
\chi_{c}(\neg \varphi)=\mathbf{L}^{n}-\chi_{c}(\varphi) .
$$


Proof. To prove (1) first observe that if $\varphi$ and $\varphi^{\prime}$ are formulas with free variables $\left(X_{1}, \ldots, X_{n}\right)$, and $\chi_{c}(\varphi)=\chi_{c}(\mathcal{A})$ and $\chi_{c}\left(\varphi^{\prime}\right)=\chi_{c}\left(\mathcal{A}^{\prime}\right)$ with $\mathcal{A}$ and $\mathcal{A}^{\prime}$ Galois stratifications of $\mathbf{A}_{k}^{n}$ with disjoint support, then $\chi_{c}\left(\varphi \vee \varphi^{\prime}\right)=\chi_{c}(\varphi)+\chi_{c}\left(\varphi^{\prime}\right)$. Hence we have

$$
\begin{gathered}
\chi_{c}\left(\varphi \vee \varphi^{\prime}\right)=\chi_{c}\left(\varphi \wedge \varphi^{\prime}\right)+\chi_{c}\left(\neg \varphi \wedge \varphi^{\prime}\right)+\chi_{c}\left(\varphi \wedge \neg \varphi^{\prime}\right), \\
\chi_{c}(\varphi)=\chi_{c}\left(\varphi \wedge \varphi^{\prime}\right)+\chi_{c}\left(\varphi \wedge \neg \varphi^{\prime}\right)
\end{gathered}
$$

and

$$
\chi_{c}\left(\varphi^{\prime}\right)=\chi_{c}\left(\varphi \wedge \varphi^{\prime}\right)+\chi_{c}\left(\neg \varphi \wedge \varphi^{\prime}\right),
$$

whence the statement follows. (2) and (3) are proven by taking the product and the complement (in the obvious sense), respectively, of the corresponding Galois stratifications, while (4) is just evident.

3.5. New invariants of formulas. Every ring morphism

$$
K_{0}^{v}\left(\operatorname{Mot}_{k, \overline{\mathbf{Q}}}\right)_{\mathbf{Q}} \longrightarrow R
$$

composed with $\chi_{c}:$ Form $_{k} \rightarrow K_{0}^{v}\left(\operatorname{Mot}_{k, \overline{\mathbf{Q}}}\right)_{\mathbf{Q}}$ will give rise to new invariants of formulas with coefficients in $k$. Let us give some examples. They all come from realization functors. Let $H^{\cdot}$ be a cohomology theory on the category of smooth projective varieties over $k$ with values in a field containing $\mathbf{Q}$. Then the realization of a motive $(S, p, n)$ in $\operatorname{Mot}_{k, \overline{\mathbf{Q}}}$ is just $p\left(H^{\cdot}\right) \otimes H^{2}\left(\mathbf{P}_{k}^{1}\right)^{\otimes n}$, with $p\left(H^{\cdot}\right)$ the image of the projector $p$ acting on cohomology. If one takes for $H^{\cdot}$ Betti or de Rham cohomology, taking the alternating sum of the ranks of the cohomology groups gives rise to the Euler characteristic Eu $: K_{0}\left(\operatorname{Mot}_{k, \overline{\mathbf{Q}}}\right) \rightarrow \mathbf{Z}$ and, after tensoring with $\mathbf{Q}$, to a morphism Eu : $K_{0}\left(\operatorname{Mot}_{k, \overline{\mathbf{Q}}}\right) \otimes \mathbf{Q} \rightarrow \mathbf{Q}$, which restricts to a morphism $\mathrm{Eu}: K_{0}^{v}\left(\operatorname{Mot}_{k, \overline{\mathbf{Q}}}\right)_{\mathbf{Q}} \rightarrow \mathbf{Q}$. Hence every formula in Form $_{k}$ has an Euler characteristic in $\mathbf{Q}$.

By taking the Hodge realization, one associates to a motive $M=(S, p, n)$ in $\operatorname{Mot}_{k, \overline{\mathbf{Q}}}$ its Hodge polynomial

$$
\operatorname{Hodge}(M)=\sum_{i, j} h^{i, j}\left(p\left(H^{\cdot}(S)\right)\right) u^{i} v^{j}(u v)^{n}
$$

in $\mathbf{Z}\left[u, v,(u v)^{-1}\right]$, with $h^{i, j}$ the rank of the $(i, j)$-part of $p\left(H^{\cdot}\right)$. So we have a ring morphism Hodge : $K_{0}\left(\operatorname{Mot}_{k, \overline{\mathbf{Q}}}\right) \otimes \mathbf{Q} \rightarrow \mathbf{Q}\left[u, v,(u v)^{-1}\right]$, which restricts to a morphism Hodge : $K_{0}^{v}\left(\operatorname{Mot}_{k, \overline{\mathbf{Q}}}\right)_{\mathbf{Q}} \rightarrow \mathbf{Q}[u, v]$. In this way one associates to every formula in Form $_{k}$ canonical Hodge numbers in $\mathbf{Q}$.

We already considered in 3.3 the Grothendieck group $K_{0}\left(\overline{\mathbf{Q}}_{\ell}, G_{k}\right)$ of the abelian category of finite dimensional $\overline{\mathbf{Q}}_{\ell}$-vector spaces with continuous $G_{k}$-action, and the morphism

$$
\mathrm{É}_{\ell}: K_{0}^{v}\left(\operatorname{Mot}_{k, \overline{\mathbf{Q}}}\right) \otimes \mathbf{Q} \longrightarrow K_{0}\left(\overline{\mathbf{Q}}_{\ell}, G_{k}\right) \otimes \mathbf{Q}
$$

induced by étale $\ell$-adic realization. Hence to every formula in $\mathrm{Form}_{k}$ is associated a canonical virtual Galois representation with rational coefficients in $K_{0}\left(\overline{\mathbf{Q}}_{\ell}, G_{k}\right) \otimes \mathbf{Q}$. 
3.6. When $k$ is of finite type over $\mathbf{Q}$, one has the following proposition.

3.6.1. Proposition. Let $R$ be a normal domain of finite type over $\mathbf{Z}$, with fraction field $k$. Let $\varphi$ be a formula in the first order language of rings with coefficients in $R$. There exists a nonzero element $f$ in $R$ such that, for every closed point $x$ of $\operatorname{Spec} R_{f}$,

$$
\operatorname{Tr} \operatorname{Frob}_{x}\left(\chi_{c}(\varphi)\right)=\operatorname{card} Z\left(\varphi, x, \mathbf{F}_{x}\right) .
$$

Proof. The proof follows directly from Proposition 3.3.1.

3.6.2. Remark. The fact that the number of points of definable sets in finite fields may be expressed as a Q-linear combination of a number of points of varieties goes back to 22 . For further results on the number of points of definable sets in finite fields, see [4] and [17].

3.7. Grothendieck groups of first order theories. This subsection is not used in the rest of the paper and its reading requires some mild familiarity with the basic language of Model Theory.

Let $\mathcal{L}$ be a first order language and let $T$ be a theory in the language $\mathcal{L}$.

We denote by $K_{0}(T)$ the quotient of the free abelian group generated by symbols $[\varphi]$ for $\varphi$ a formula in $\mathcal{L}$ by the subgroup generated by the following relations:

(1) If $\varphi$ is a formula in $\mathcal{L}$ with free variables $x=\left(x_{1}, \ldots, x_{n}\right)$ and $\varphi^{\prime}$ is a formula in $\mathcal{L}$ with free variables $x^{\prime}=\left(x_{1}^{\prime}, \ldots, x_{n^{\prime}}^{\prime}\right)$, then $[\varphi]=\left[\varphi^{\prime}\right]$ if there exists a formula $\psi$ in $\mathcal{L}$, with free variables $\left(x, x^{\prime}\right)$, such that

$$
\begin{aligned}
& T \models\left[\forall x\left(\varphi(x) \rightarrow \exists ! x^{\prime}:\left(\varphi^{\prime}\left(x^{\prime}\right) \wedge \psi\left(x, x^{\prime}\right)\right)\right)\right] \\
& \wedge {\left[\forall x^{\prime}\left(\varphi^{\prime}\left(x^{\prime}\right) \rightarrow \exists ! x:\left(\varphi(x) \wedge \psi\left(x, x^{\prime}\right)\right)\right)\right] . }
\end{aligned}
$$

(2) $\left[\varphi \vee \varphi^{\prime}\right]=[\varphi]+\left[\varphi^{\prime}\right]-\left[\varphi \wedge \varphi^{\prime}\right]$, for $\varphi$ and $\varphi^{\prime}$ formulas in $\mathcal{L}$.

Furthermore one puts a ring structure on $K_{0}(T)$ by setting

(3) $[\varphi(x)] \cdot\left[\varphi^{\prime}\left(x^{\prime}\right)\right]=\left[\varphi(x) \wedge \varphi^{\prime}\left(x^{\prime}\right)\right]$, if $\varphi$ and $\varphi^{\prime}$ are formulas in $\mathcal{L}$ with disjoint free variables $x$ and $x^{\prime}$.

For every interpretation of a theory $T_{1}$ in a theory $T_{2}$ there is a canonical morphism of rings $K_{0}\left(T_{1}\right) \rightarrow K_{0}\left(T_{2}\right)$, and this gives rise to a functor from the category of theories in $\mathcal{L}$, morphisms being given by interpretation, to the category of commutative rings.

The previous constructions may now be rephrased in the following way.

3.7.1. Theorem. Let $k$ be a field of characteristic zero. Let $\mathcal{L}$ be the first order language of rings with coefficients in $k$ and let $T$ be the theory of pseudo-finite fields containing $k$. There exists a canonical morphism of rings

$$
\chi_{c}: K_{0}(T) \longrightarrow K_{0}^{v}\left(\operatorname{Mot}_{k, \overline{\mathbf{Q}}}\right)_{\mathbf{Q}}
$$

factorizing the morphism

$$
\chi_{c}: K_{0}\left(\operatorname{Sch}_{k}\right) \longrightarrow K_{0}^{v}\left(\operatorname{Mot}_{k, \overline{\mathbf{Q}}}\right)_{\mathbf{Q}}
$$

Proof. Indeed, this follows from Proposition 3.4 .3 and Proposition 3.4.4

3.7.2. Remark. If $k$ is a field and $T_{\mathrm{ac}}$ is the theory of algebraically closed fields containing $k$, then $K_{0}\left(T_{\mathrm{ac}}\right)$ is isomorphic to $K_{0}\left(\mathrm{Sch}_{k}\right)$. If $T_{\mathbf{R}}$ is the theory of real closed fields in the language of ordered rings, then $K_{0}\left(T_{\mathbf{R}}\right)$ is isomorphic to $\mathbf{Z}$. 
Recently, Cluckers and Haskell [5] proved that the theory of any fixed $p$-adic field, in the language of rings, has trivial Grothendieck group.

\section{Definable Subassignments For Rings}

4.1. Let $h: \mathcal{C} \rightarrow$ Sets be a functor from a category $\mathcal{C}$ to the category of sets. We shall call the data for each object $C$ of $\mathcal{C}$ of a subset $h^{\prime}(C)$ of $h(C)$ a subassignment of $h$. The point in this definition is that $h^{\prime}$ is not assumed to be a subfunctor of $h$.

For $h^{\prime}$ and $h^{\prime \prime}$ two subassignments of $h$, we shall denote by $h^{\prime} \cap h^{\prime \prime}$ and $h^{\prime} \cup h^{\prime \prime}$ the subassignments $C \mapsto h^{\prime}(C) \cap h^{\prime \prime}(C)$ and $C \mapsto h^{\prime}(C) \cup h^{\prime \prime}(C)$, respectively. Similarly, we denote by $h \backslash h^{\prime}$ the subassignment $C \mapsto h(C) \backslash h^{\prime}(C)$. We also write $h \subset h^{\prime}$ if $h(C) \subset h^{\prime}(C)$ for every object $C$ of $\mathcal{C}$.

If $h^{\prime}$ is a subassignment of a functor $h$, and $\pi: h \rightarrow h_{0}$ is a natural transformation of functors, then we denote by $\pi\left(h^{\prime}\right)$ the subassignment of $h_{0}$ given by $C \mapsto \pi\left(h^{\prime}(C)\right)$.

Let $R$ be a ring. We denote by Field ${ }_{R}$ the category of fields which are $R$-algebras. For $X$ a variety over Spec $R$, we consider the functor $h_{X}: k \mapsto X(k)$ from Field ${ }_{R}$ to the category of sets.

Let $\varphi\left(X_{1}, \ldots, X_{m}\right)$ be a formula in the first order language of rings with coefficients in $R$ and free variables $X_{1}, \ldots, X_{m}$ and let $\mathbf{A}_{R}^{m}$ be the affine space Spec $R\left[X_{1}, \ldots, X_{m}\right]$. For every field $k$ in Field $_{R}$, we denote by $Z(\varphi, k)$ the subset of $k^{m}=\mathbf{A}_{R}^{m}(k)$ defined by the formula $\varphi$. This gives rise to a subassignment $k \mapsto Z(\varphi, k)$ of the functor $h_{\mathbf{A}_{R}^{m}}$. We call such a subassignment a definable subassignment of $h_{\mathbf{A}_{R}^{m}}$. Let $X$ be a variety over $\operatorname{Spec} R$. Assume first $X$ is affine and embedded as a closed subscheme in $\mathbf{A}_{R}^{m}$. We shall say a subassignment of $h_{X}$ is a definable subassignment if it is a definable subassignment of $h_{\mathbf{A}_{R}^{m}}$. Clearly, this definition is independent of the choice of the embedding of $X$ in an affine space. In general, a subassignment $h$ of $h_{X}$ will be said to be definable if there exists a finite cover $\left(X_{i}\right)_{i \in I}$ of $X$ by affine open subschemes and definable subassignments $h_{i}$ of $h_{X_{i}}$, for $i \in I$, such that $h=\bigcup_{i \in I} h_{i}$. When $X$ is affine this definition coincides with the previous one.

We shall denote by $\operatorname{Def}_{R}(X)$ the set of definable subassignments of $h_{X}$. Clearly $\operatorname{Def}_{R}(X)$ is stable by finite intersection and finite union and by taking complements.

4.2. Now let $k$ be a field of characteristic zero. Assume first $X$ is a closed affine subvariety of $\mathbf{A}_{k}^{m}$ and let $h$ be a definable subassignment of $h_{X}$ associated to a formula $\varphi$ with coefficients in $k$ and $m$ free variables. We set $\chi_{c}(h)=\chi_{c}(\varphi)$. It follows from Proposition 3.4 .3 that $\chi_{c}(h)$ is independent of $\varphi$ and the embedding of $X$. In general, when $X$ is a variety over $k$ and $h$ is a definable subassignment of $h_{X}$, one takes a finite cover $\left(X_{i}\right)_{i \in I}$ of $X$ by affine open subschemes and definable subassignments $h_{i}$ of $h_{X_{i}}$ as in 4.1 and one sets

$$
\chi_{c}(h)=\sum_{\emptyset \neq J \subset I} \chi_{c}\left(\bigcap_{i \in J} h_{X_{i}} \backslash \bigcup_{i \notin J} h_{X_{i}}\right)
$$

which is well defined by Proposition 3.4.3 and Proposition 3.4.4. It also follows from Proposition 3.4.4 that $\chi_{c}$ is additive, i.e. that $\chi_{c}\left(h \cup h^{\prime}\right)=\chi_{c}(h)+\chi_{c}\left(h^{\prime}\right)-\chi_{c}\left(h \cap h^{\prime}\right)$.

4.2.1. Remark. Of course we could also have defined $\chi_{c}(h)$ for $h$ a definable subassignment of $h_{X}$ by directly associating a Galois stratification to $h$, without considering formulas. 
4.3. Let $R$ be a normal domain of finite type over $\mathbf{Z}$, with fraction field $k$. Let $X$ be a variety over $R$ and let $h$ be a definable subassignment of $h_{X}$. We shall denote by $h \otimes k$ the definable subassignment of $h_{X \otimes k}$ obtained by extension of scalars.

The following result follows directly from Proposition 3.6.1

4.3.1. Proposition. Let $R$ be a normal domain of finite type over $\mathbf{Z}$, with fraction field $k$. Let $X$ be a variety over $R$ and let $h$ be a definable subassignment of $h_{X}$. There exists a nonzero element $f$ in $R$ such that, for every closed point $x$ of $\operatorname{Spec} R_{f}$,

$$
\operatorname{Tr} \operatorname{Frob}_{x}\left(\chi_{c}(h \otimes k)\right)=\operatorname{card} h\left(\mathbf{F}_{x}\right) .
$$

4.4. Let $R$ be a ring and let $X$ be an $R$-variety. Let $h$ and $h^{\prime}$ be definable subassignments of $h_{X}$. We shall write $h \approx h^{\prime}$ if $h(k)=h^{\prime}(k)$ for every $k$ in Field $_{R}$ which is a pseudo-finite field. More generally, if $X$ and $X^{\prime}$ are $R$-varieties, and $h$ and $h^{\prime}$ are definable subassignments of $h_{X}$ and $h_{X^{\prime}}$, respectively, we write $h \equiv h^{\prime}$ if there exists a definable subassignment $h^{\prime \prime}$ of $h_{X \otimes X^{\prime}}$ such that, for every field $k$ in Field ${ }_{R}$ which is a pseudo-finite field, $h^{\prime \prime}(k)$ is the graph of a bijection between $h(k)$ and $h^{\prime}(k)$. Clearly $\approx$ and $\equiv$ are equivalence relations.

Let $R$ be a normal domain and let $X$ be a variety over $R$. Let $\mathcal{A}$ be a Galois stratification of $X$. We can associate to $\mathcal{A}$ a subassignment $h_{\mathcal{A}}$ of $h_{X}$ by defining, for $k$ in Field $_{R}$, as in 2.2

$$
h_{\mathcal{A}}(k):=\left\{\mathbf{a}=\left(a_{1}, \ldots, a_{n}\right) \in X(k) \mid \operatorname{Ar}(\mathbf{a}) \subset \operatorname{Con}(\mathcal{A})\right\} .
$$

We shall call such subassignments Galois subassignments of $h_{X}$.

In this language Corollary 2.3.2 can be reformulated as follows.

4.4.1. Proposition. Let $k$ be a field and let $X$ be a variety over $k$. Let $h$ be a definable subassignment of $h_{X}$. Then there exists a Galois subassignment $h^{\prime}$ of $h_{X}$ such that $h \approx h^{\prime}$.

The following result follows directly from Proposition 3.4 .3

4.4.2. Proposition. Let $k$ be a field and let $X$ and $X^{\prime}$ be $k$-varieties. If $h$ and $h^{\prime}$ are definable subassignments of $h_{X}$ and $h_{X^{\prime}}$, respectively, such that $h \equiv h^{\prime}$, then $\chi_{c}(h)=\chi_{c}\left(h^{\prime}\right)$.

4.5. Let $h$ be a subassignment of $h_{X}$ for $X$ a variety over $k$. We say that $h$ is of dimension $\leq r$ if there exists a closed subvariety $S$ of $X$ of dimension $\leq r$ such that $h$ is a subassignment of $h_{S}$.

\section{Definable SUbASSIGNMENTS FOR POWER SERIES RINGS}

5.1. Quantifier elimination for valued fields. Let $K$ be a valued field, with valuation ord : $K \rightarrow \Gamma \cup\{\infty\}$, where $\Gamma$ is an ordered abelian group. We denote by $\mathcal{O}_{K}$ the valuation ring, by $P$ the valuation ideal, by $U$ the group of units in $\mathcal{O}_{K}$, by $\kappa$ the residue field, and by Res : $\mathcal{O}_{K} \rightarrow \kappa$ the canonical projection. We assume that $K$ has an angular component map. By this we mean a map $\overline{\mathrm{ac}}: K \rightarrow \kappa$ such that $\overline{\mathrm{ac}} 0=0$, the restriction of $\overline{\mathrm{ac}}$ to $K^{\times}$is multiplicative and the restriction of $\overline{\mathrm{ac}}$ to $U$ coincides with the restriction of Res. From now on we fix that angular component map $\overline{\mathrm{ac}}$.

We consider 3 -sorted first order language 3 of the form

$$
\mathcal{L}=\left(\mathbf{L}_{K}, \mathbf{L}_{\kappa}, \mathbf{L}_{\Gamma}, \text { ord }, \overline{\mathrm{ac}}\right),
$$

\footnotetext{
${ }^{3}$ See, e.g., [15], pp. 277-281, for more information concerning many-sorted first order logic.
} 
consisting of

(i) the language $\mathbf{L}_{K}=\{+,-, \times, 0,1\}$ of rings as valued field sort,

(ii) the language $\mathbf{L}_{\kappa}=\{+,-, \times, 0,1\}$ of rings as residue field sort,

(iii) a language $\mathbf{L}_{\Gamma}$, which is an extension of the language $\{+, 0, \infty, \leq\}$ of ordered abelian groups with an element $\infty$ on top, as the value sort,

(iv) a function symbol ord from the valued field sort to the value sort, which stands for the valuation,

(v) a function symbol $\overline{\mathrm{ac}}$ from the valued field sort to the residue field sort, which stands for the angular component map.

In the following we shall assume that $K$ is henselian and that $\kappa$ is of characteristic zero. We consider $(K, \kappa, \Gamma \cup\{\infty\}$, ord, $\overline{\mathrm{ac}})$ as a structure for the language $\mathcal{L}$, the interpretations of symbols being the standard ones. By a henselian $\mathcal{L}$-extension of $K$, we mean an extension $\left(K^{\prime}, \kappa^{\prime}, \Gamma^{\prime} \cup\{\infty\}\right.$, $\left.\operatorname{ord}^{\prime}, \overline{\mathrm{ac}}^{\prime}\right)$ of the structure $(K, \kappa, \Gamma \cup$ $\{\infty\}$, ord, $\overline{\mathrm{ac}})$ with respect to the language $\mathcal{L}$, with $K^{\prime}$ a henselian valued field. (By an extension, we mean a structure for the language $\mathcal{L}$ which contains the original structure as a substructure.) By abuse of language we shall say that $K^{\prime}$ is a henselian $\mathcal{L}$-extension of $K$.

We may now state the quantifier elimination theorem of Pas [26].

5.1.1. Theorem. Let $K$ be a valued field which satisfies the previous conditions. For every $\mathcal{L}$-formula $\varphi$ there exists an $\mathcal{L}$-formula $\varphi^{\prime}$ without quantifiers over the valued field sort such that $\varphi$ is equivalent in $K^{\prime}$ to $\varphi^{\prime}$, for every henselian $\mathcal{L}$-extension $K^{\prime}$ of $K$.

Proof. This follows from Theorem 4.1 of 26 together with the observation at the begining of section 3 of [26].

In particular, when the value group is $\mathbf{Z}$, we shall use the language

$$
\mathcal{L}_{\mathrm{Pas}}=\left(\mathbf{L}_{K}, \mathbf{L}_{\kappa}, \mathbf{L}_{\mathrm{PR} \infty}, \text { ord }, \overline{\mathrm{ac}}\right),
$$

where $\mathbf{L}_{\mathrm{PR} \infty}=\mathbf{L}_{\mathrm{PR}} \cup\{\infty\}$ and $\mathbf{L}_{\mathrm{PR}}$ is the Presburger language

$$
\mathbf{L}_{\mathrm{PR}}=\{+, 0,1, \leq\} \cup\left\{\equiv_{n} \mid n \in \mathbf{N}, n>1\right\},
$$

where $\equiv_{n}$ will be interpreted as "congruent modulo $n$ " in $\Gamma$. We call a subset of $\mathbf{Z}^{n}$ which is definable in the language $\mathbf{L}_{\mathrm{PR}}$ a Presburger subset of $\mathbf{Z}^{n}$. Similarly, we call a function $\mathbf{Z}^{m} \rightarrow \mathbf{Z}^{r}$ a Presburger function if its graph is definable in $\mathbf{L}_{\mathrm{PR}}$.

5.1.2. Corollary. Let $K$ be a valued field with value group $\Gamma$ elementary equivalen 4 to $\mathbf{Z}$ in the language of ordered abelian groups and satisfying the previous conditions for $\mathcal{L}$ replaced by the language $\mathcal{L}_{\text {Pas }}$. For every $\mathcal{L}_{\text {Pas }}$-formula $\varphi$ there exists an $\mathcal{L}_{\mathrm{Pas}}$-formula $\varphi^{\prime}$ without quantifiers over the valued field sort and the value sort such that $\varphi$ is equivalent in $K^{\prime}$ to $\varphi^{\prime}$, for every henselian $\mathcal{L}_{\text {Pas }}$-extension $K^{\prime}$ of $K$ with value group elementary equivalent to $\mathbf{Z}$.

Proof. Indeed, it follows from Theorem 5.1.1, since, by a classical result of Presburger [27, $\mathbf{Z}$ has quantifier elimination in the language $\mathbf{L}_{\mathrm{PR}}$.

\footnotetext{
${ }^{4}$ See, e.g., [18 for this notion.
} 
5.2. We assume from now on that $K=k((t))$, that $\kappa=k$, with $k$ a field of characteristic zero, and that ord and $\overline{\mathrm{ac}}$ have their classical meaning for formal power series: if $\varphi$ belongs to $k((t))$, ord $(\varphi)$ will denote the order in $t$ of $\varphi$ and $\overline{\operatorname{ac}}(\varphi)$ the coefficient of $t^{\operatorname{ord}(\varphi)}$ in $\varphi$, with the convention $\overline{\mathrm{ac}}(0)=0$. In particular, the hypotheses of Corollary 5.1 .2 are satisfied in the language $\mathcal{L}_{\text {Pas }}$.

Let $R$ be a subring of $k$. By an $\mathcal{L}_{\text {Pas }}$-formula with coefficients in $R$ in the valued field sort and in the residue field sort we mean a formula in the language obtained from $\mathcal{L}_{\text {Pas }}$ by adding, for every element of $R$, a new symbol to denote it in the valued field sort and in the residue field sort. Note that Corollary 5.1.2 remains valid for such formulas. We shall consider $\mathcal{L}_{\text {Pas }}$-formulas with coefficients in $R$ in the valued field sort and in the residue field sort, free variables $x_{1}, \ldots, x_{m}$ running over the valued field sort and no free variables running over the residue field or the value sort. We shall call such formulas formulas on $R[[t]]^{m}$. The reason for that denomination is that later we shall view the free variables $x_{1}, \ldots, x_{m}$ as running over $R[[t]]$. More generally, an $\mathcal{L}_{\text {Pas }}$-formula with coefficients in $R$ in the valued field sort and in the residue field sort, free variables $x_{1}, \ldots, x_{m}$ running over the valued field sort, no free variables running over the residue field sort and $r$ free variables running over the value sort give rise by specialization of the value sort variables to $\mathbf{Z}^{r}$ (resp. $\mathbf{N}^{r}$ or $\mathbf{N} \cup\{\infty\}$ when $r=1$ ) to what we shall call a formula on $R[[t]]^{m}$ depending on parameters in $\mathbf{Z}^{r}$ (resp. $\mathbf{N}^{r}$ or $\mathbf{N} \cup\{\infty\}$ ).

We shall deduce the following statement of Ax/Ax-Kochen-Eršov type from the Theorem of Pas.

5.2.1. Proposition. Let $R$ be a normal domain of finite type over $\mathbf{Z}$ with field of fractions $k$. Let $\sigma$ be a sentence in the language $\mathcal{L}_{\text {Pas }}$ with coefficients in $R$ in the valued field sort and in the residue field sort. The following statements are equivalent:

(1) The sentence $\sigma$ is true in $F((t))$ for every pseudo-finite field $F$ containing $k$.

(2) There exists $f$ in $R \backslash\{0\}$ such that, for every closed point $x$ in $\operatorname{Spec} R_{f}$, the sentence $\sigma$ is true in $\mathbf{F}_{x}((t))$.

If, furthermore, $k$ is a finite extension of $\mathbf{Q}$, the previous statements are also equivalent to the following:

(3) There exists $f$ in $R \backslash\{0\}$, multiple of the discriminant of $k / \mathbf{Q}$, such that, for every closed point $x$ in $\operatorname{Spec} R_{f}$, the sentence $\sigma$ is true in $k_{x}$,

where $k_{x}$ denotes the completion of $k$ at $x$. Remark that, the extension $k / \mathbf{Q}$ being nonramified at $x$, the field $k_{x}$ admits a canonical uniformizing parameter, hence also a canonical angular component map.

Proof. Let us first prove the equivalence of (1) and (2). By Corollary 5.1 .2 there exists an $\mathcal{L}_{\text {Pas }}$-sentence $\sigma^{\prime}$ without quantifiers over the valued field sort and the value sort such that $\sigma$ is equivalent in $K^{\prime}$ to $\sigma^{\prime}$, for every henselian $\mathcal{L}_{\text {Pas }}$-extension $K^{\prime}$ of $k((t))$. Hence there exists $f$ in $R \backslash\{0\}$ such that, for every closed point $x$ in Spec $R_{f}$, the sentence $\sigma$ is equivalent to $\sigma^{\prime}$ in $\mathbf{F}_{x}((t))$. Indeed, if this would not be the case, a suitable ultraproduct of the fields $\mathbf{F}_{x}((t))$ would yield a henselian $\mathcal{L}_{\text {Pas }}$-extension $K^{\prime}$ of $k((t))$ in which $\sigma$ would not be equivalent to $\sigma^{\prime}$. Hence we may assume $\sigma$ is a sentence (i.e. is without free variables) and has quantifiers only over the residue field sort, in which case the result follows from Proposition 2.4.2 The proof of the equivalence of (1) and (3) is completely similar. 
5.3. We shall call a formula on $R[[t]]^{m}$ a special formula on $R[[t]]^{m}$ if it is obtained by repeated application of conjunction and negation from formulas of the form

$$
\begin{gathered}
\operatorname{ord} f_{1}\left(x_{1}, \ldots, x_{m}\right) \geq \operatorname{ord} f_{2}\left(x_{1}, \ldots, x_{m}\right)+a, \\
\operatorname{ord} f_{1}\left(x_{1}, \ldots, x_{m}\right) \equiv a \bmod b
\end{gathered}
$$

and

$$
\vartheta\left(\overline{\operatorname{ac}}\left(f_{1}\left(x_{1}, \ldots, x_{m}\right)\right), \ldots, \overline{\operatorname{ac}}\left(f_{m^{\prime}}\left(x_{1}, \ldots, x_{m}\right)\right)\right),
$$

where the $f_{i}$ are polynomials with coefficients in $R, a$ and $b$ are in $\mathbf{Z}$, and $\vartheta$ is a formula in $m^{\prime}$ free variables in the first order language of rings with coefficients in $R$.

Replacing $a$ in the above formulas by $L\left(a_{1}, \ldots, a_{r}\right)$ with $L$ a polynomial with coefficients in $\mathbf{Z}$ and degree $\leq 1$, one gets the definition of special formulas on $R[[t]]^{m}$ depending on parameters in $\mathbf{Z}^{r}$ (resp. $\mathbf{N}^{r}$ or $\mathbf{N} \cup\{\infty\}$ ).

5.4. Let $k$ be a field. For $X$ a variety over $k$, we will denote by $\mathcal{L}(X)$ the scheme of germs of arcs on $X$. It is a scheme over $k$ and, for every field extension $k \subset K$, there is a natural bijection,

$$
\mathcal{L}(X)(K) \simeq \operatorname{Mor}_{k \text {-schemes }}(\operatorname{Spec} K[[t]], X),
$$

between the set of $K$-rational points of $\mathcal{L}(X)$ and the set of germs of arcs with coefficients in $K$ on $X$. We will call the $K$-rational points of $\mathcal{L}(X)$, for $K$ a field extension of $k$, arcs on $X$, and $\varphi(0)$ will be called the origin of the $\operatorname{arc} \varphi$. More precisely, the scheme $\mathcal{L}(X)$ is defined as the projective limit,

$$
\mathcal{L}(X):=\varliminf_{\longleftarrow} \mathcal{L}_{n}(X),
$$

in the category of $k$-schemes of the schemes $\mathcal{L}_{n}(X)$ representing the functor

$$
R \mapsto \operatorname{Mor}_{k \text {-schemes }}\left(\operatorname{Spec} R[t] / t^{n+1} R[t], X\right),
$$

defined on the category of $k$-algebras. (The existence of $\mathcal{L}_{n}(X)$ is well known (cf. [12]), and the projective limit exists since the transition morphisms are affine.) We shall denote by $\pi_{n}$ the canonical morphism corresponding to truncation of arcs,

$$
\pi_{n}: \mathcal{L}(X) \longrightarrow \mathcal{L}_{n}(X) .
$$

The schemes $\mathcal{L}(X)$ and $\mathcal{L}_{n}(X)$ will always be considered to have their reduced structure.

5.5. Let $R$ be a ring and let $X$ be a variety over $\operatorname{Spec} R$. We consider the functor $h_{\mathcal{L}(X)}: k \mapsto X(k[[t]])$ from Field $_{R}$ to the category of sets.

Let $\varphi$ be a formula on $R[[t]]^{m}$. For every field $k$ in Field $R$, denote by $Z(\varphi, k[[t]])$ the subset of all $x$ in $k[[t]]^{m}=\mathbf{A}_{R}^{m}(k[[t]])$ for which $\varphi(x)$ is true in $k((t))$. This defines a subassignment $k \mapsto Z(\varphi, k[[t]])$ of the functor $h_{\mathcal{L}\left(\mathbf{A}_{R}^{m}\right)}$. We call such a subassignment a definable subassignment of $h_{\mathcal{L}\left(\mathbf{A}_{R}^{m}\right)}$. We now proceed in a similar way as in 4.1 to define definable subassignments of $h_{\mathcal{L}(X)}$, for $X$ a variety over Spec $R$. When $X$ is affine and embedded as a closed subscheme in $\mathbf{A}_{R}^{m}$, we shall say a subassignment of $h_{\mathcal{L}(X)}$ is a definable subassignment if it is a definable subassignment of $h_{\mathcal{L}\left(\mathbf{A}_{R}^{m}\right)}$. In general a subassignment $h$ of $h_{\mathcal{L}(X)}$ will be said to be definable if there exists a finite cover $\left(X_{i}\right)_{i \in I}$ of $X$ by affine open subschemes and definable subassignments $h_{i}$ of $h_{\mathcal{L}\left(X_{i}\right)}$ such that $h=\bigcup_{i \in I} h_{i}$. Similarly, one defines 
definable subassignments $h_{a}$ of $h_{\mathcal{L}(X)}$ depending on parameters $a=\left(a_{1}, \ldots, a_{r}\right)$ in $\mathbf{Z}^{r}$, in $\mathbf{N}^{r}$, or in $\mathbf{N} \cup\{\infty\}$.

We shall denote by $\operatorname{Def}_{R}(\mathcal{L}(X))$ the set of definable subassignments of $h_{\mathcal{L}(X)}$. Clearly $\operatorname{Def}_{R}(\mathcal{L}(X))$ is stable by finite intersection and finite union and by taking complements.

We shall use the symbol $\dot{\cup}$ to denote finite or infinite union of pairwise disjoint subassignments of $h_{\mathcal{L}(X)}$.

For a ring morphism $R \rightarrow R^{\prime}$ and $h$ a functor or a subassignment from Field ${ }_{R}$ to the category of sets, we denote by $h \otimes R^{\prime}$ the restriction of $h$ to Field $R_{R^{\prime}}$.

The following statement is a direct consequence of Corollary 5.1.2.

5.5.1. Proposition. Let $k$ be a field of characteristic zero. Let $\varphi$ be a formula (resp. a formula depending on parameters) on $k[t]]^{m}$. Then there exists a special formula (resp. a special formula depending on parameters) $\varphi^{\prime}$ on $k[[t]]^{m}$ such that $\varphi$ and $\varphi^{\prime}$ define the same definable subassignment (resp. the same definable subassignment depending on parameters) of $h_{\mathcal{L}\left(\mathbf{A}_{k}^{m}\right)}$.

5.6. Let $k$ be a field and let $X$ be a $k$-variety. Let $h$ be a definable subassignment of $h_{\mathcal{L}(X)}$. By a definable partition of $h$, with parameters in $\mathbf{Z}, \mathbf{N}$, or $\mathbf{N} \cup\{\infty\}$, we mean the data of definable subassignments $h_{i}$, depending on the parameter $i \in \mathbf{Z}$, $\mathbf{N}$, or $\mathbf{N} \cup\{\infty\}$, which are pairwise disjoint and such that $h=\bigcup_{i} h_{i}$. Similarly, if $h_{n}$ already depends on a parameter $n$, a definable partition of the subassignments $h_{n}$, with parameters in $\mathbf{Z}, \mathbf{N}$, or $\mathbf{N} \cup\{\infty\}$, will be the data of definable subassignments $h_{n, i}$, depending on the parameter $(n, i)$, such that, for each $n$, the subassignments $h_{n, i}$ are pairwise disjoint and $h_{n}=\bigcup_{i} h_{n, i}$.

5.7. Truncation of definable subassignments. Let $k$ be a field of characteristic zero and let $X$ be a variety over $k$. For $n$ in $\mathbf{N}$, we have a canonical truncation morphism $\pi_{n}: \mathcal{L}(X) \rightarrow \mathcal{L}_{n}(X)$. Hence if $h$ is a subassignment of $h_{\mathcal{L}(X)}$ (resp. of $\left.h_{\mathcal{L}_{n}(X)}\right)$ we may consider $\pi_{n}(h): K \mapsto \pi_{n}(h(K))\left(\right.$ resp. $\left.\pi_{n}^{-1}(h): K \mapsto \pi_{n}^{-1}(h(K))\right)$ which is a subassignment of $h_{\mathcal{L}_{n}(X)}\left(\right.$ resp. of $\left.h_{\mathcal{L}(X)}\right)$.

5.7.1. Proposition. Let $h$ be a definable subassignment of $h_{\mathcal{L}(X)}$. Then, for every $n$ in $\mathbf{N}, \pi_{n}(h)$ is a definable subassignment of $h_{\mathcal{L}_{n}(X)}$ in the sense of section $\square$ and $\pi_{n}^{-1} \pi_{n}(h)$ is a definable subassignment of $h_{\mathcal{L}(X)}$.

Proof. One may assume $X=\mathbf{A}_{k}^{m}$ and $h$ is associated to a formula $\varphi$ on $k[[t]]^{m}$. Then $\pi_{n}^{-1} \pi_{n}(h)$ is associated to the formula

$$
\exists y_{1} \ldots \exists y_{m}\left(\left(\operatorname{ord}\left(x_{1}-y_{1}\right)>n\right) \wedge \cdots \wedge\left(\operatorname{ord}\left(x_{m}-y_{m}\right)>n\right) \wedge \varphi\left(y_{1}, \ldots, y_{m}\right)\right)
$$

and it follows from Corollary 5.1.2 that $\pi_{n}^{-1} \pi_{n}(h)$ is a definable subassignment of $h_{\mathcal{L}(X)}$ associated to a special formula on $k[[t]]^{m}$ - without quantifiers in the valued field sort - say $\psi$. One can formally write $x_{i}=\sum_{j \geq 0} a_{j}^{(i)} t^{j}$ and then, by expanding the variables $x_{i}$ into series in the formulas of type (5.3.1), (5.3.2) and (5.3.3) appearing in $\psi$, one obtains an infinite set of conditions in the variables $a_{j}^{(i)}$. If one substitutes in these conditions $a_{j}^{(i)}=0$ whenever $i>n$, then only a finite number of conditions which involve only coefficients $a_{j}^{(i)}$ with $j \leq n$ remain. In this way one obtains from the formula $\psi$ a formula $\tau_{n}(\psi)$ with coefficients in $k$ and free variables $a_{j}^{(i)}, j \leq n$, and $\pi_{n}(h)$ is the definable subassignment of $h_{\mathcal{L}_{n}(X)}$ associated to $\tau_{n}(\psi)$. 
5.8. Stable definable subassignments. Let $k$ be a field of characteristic zero and let $X$ be a variety over $k$. Let $h$ be a definable subassignment of $h_{\mathcal{L}(X)}$. We say $h$ is weakly stable at level $n$ if $h=\pi_{n}^{-1} \pi_{n}(h)$ and that $h$ is weakly stable if it is weakly stable at some level.

5.8.1. Lemma. Let $h$ and $h_{i}, i \in \mathbf{N}$, be weakly stable definable subassignments of $h_{\mathcal{L}(X)}$. Assume that

$$
h=\bigcup_{i \in \mathbf{N}} h_{i} .
$$

Then there exists a natural number $n$ such that

$$
h=\bigcup_{i \leq n} h_{i} .
$$

Proof. The proof of Lemma 2.4 in [12] using ultraproducts may be directly adapted to the present situation. Indeed, we may assume $X$ is a closed subvariety of $\mathbf{A}_{k}^{m}$, and it is enough to prove that if $k_{i}, i \in \mathbf{N}$, are weakly stable definable subassignments of $h_{\mathcal{L}(X)}$ such that, for every finite subset $\Sigma$ of $\mathbf{N}, \bigcap_{i \in \Sigma} k_{i}$ is not the empty subassignment, i.e. for some field $K_{\Sigma}, \bigcap_{i \in \Sigma} k_{i}\left(K_{\Sigma}\right)$ is not empty, then $\bigcap_{i \in \mathbf{N}} k_{i}$ is not the empty subassignment. Since every weakly stable definable subassignment of $h_{\mathcal{L}(X)}$ may be defined by an infinite conjunction of formulas in the language of rings with coefficients in $k$ each involving only a finite number of coefficients of the power series $x_{i}, 1 \leq i \leq m$ (cf. the proof of Proposition 5.7.1), it follows that $\bigcap_{i \in \mathbf{N}} k_{i}\left(K^{*}\right)$ is not empty for $K^{*}$ the ultraproduct of the fields $K_{\Sigma}$ with respect to a suitable ultrafilter.

Let $\pi: X \rightarrow Y$ be a morphism of algebraic varieties over $k$ and let $h$ and $h^{\prime}$ be definable subassignments of $h_{X}$ and $h_{Y}$, respectively. Assume $\pi(h) \subset h^{\prime}$. We say that $\pi$ induces a piecewise trivial fibration $h \rightarrow h^{\prime}$ with fiber a $k$-variety $F$, if there exists a finite family of locally closed subsets $S_{i}, i \in I$, of $Y$, such that $\pi^{-1}\left(S_{i}\right)$ is locally closed in $X$, with $h^{\prime} \subset \bigcup_{i \in I} h_{S_{i}}$, such that there is an isomorphism $\pi^{-1}\left(S_{i}\right) \simeq S_{i} \times F$, with $\pi$ corresponding under the isomorphism to the projection $S_{i} \times F \rightarrow S_{i}$, inducing, for every $L$ in Field $_{k}$, a bijection between $\left(h \cap h_{\pi^{-1}\left(S_{i}\right)}\right)(L)$ and $\left(h^{\prime} \cap h_{S_{i}}\right)(L) \times h_{F}(L)$.

Let $X$ be an algebraic variety over $k$ of dimension $d \geq 0$ and let $h$ be a definable subassignment of $h_{\mathcal{L}(X)}$. We say $h$ is stable at level $n \in \mathbf{N}$, if $h$ is weakly stable at level $n$ and the canonical morphism $\mathcal{L}_{m+1}(X) \rightarrow \mathcal{L}_{m}(X)$ induces a piecewise trivial fibration $\pi_{m+1}(h) \rightarrow \pi_{m}(h)$ with fiber $\mathbf{A}_{k}^{d}$ for every $m \geq n$.

We say $h$ is stable if it is stable at some level $n$. The set $\operatorname{Def}_{k}(\mathcal{L}(X))_{s t}$ of stable definable subassignments of $h_{\mathcal{L}(X)}$ is stable by taking finite intersections and finite unions.

5.8.2. Remark. If $h$ is a weakly stable subassignment of $h_{\mathcal{L}(X)}$ and $h \cap h_{\mathcal{L}\left(X_{0}\right)}=\emptyset$, with $X_{0}$ the union of the singular locus of $X$ and its irreducible components of dimension $<d$, then $h$ is stable, as follows from Lemma 5.8.1 and Lemma 4.1 of [12. In particular, if $X$ is smooth of pure dimension $d$, every weakly stable subassignment of $h_{\mathcal{L}(X)}$ is stable.

5.8.3. Lemma. If $h$ is stable at level $n$, then, for every $n^{\prime} \geq n$,

$$
\chi_{c}\left(\pi_{n^{\prime}}(h)\right) \mathbf{L}^{-\left(n^{\prime}+1\right) d}=\chi_{c}\left(\pi_{n}(h)\right) \mathbf{L}^{-(n+1) d} .
$$

Proof. Clear. 
5.9. Let $R$ be a ring and let $X$ be an $R$-variety. Let $h$ and $h^{\prime}$ be subassignments of $h_{\mathcal{L}(X)}$. We shall write $h \approx h^{\prime}$ if $h(k)=h^{\prime}(k)$ for every $k$ in Field $R_{R}$ which is a pseudo-finite field. If $h \approx h^{\prime}$, then $\pi_{n}(h) \approx \pi_{n}\left(h^{\prime}\right)$ for every $n$ in $\mathbf{N}$.

\section{Arithmetic motivic integration on Definable Sets}

6.1. Let $k$ be a field of characteristic 0 and let $X$ be a variety over $k$ of dimension $d$. By Lemma 5.8 .3 there exists a unique function

$$
\tilde{\nu}: \operatorname{Def}_{k}(\mathcal{L}(X))_{s t} \longrightarrow K_{0}^{v}\left(\operatorname{Mot}_{k, \overline{\mathbf{Q}}}\right)_{\mathrm{loc}, \mathbf{Q}}
$$

such that

$$
\tilde{\nu}(h)=\chi_{c}\left(\pi_{n}(h)\right) \mathbf{L}^{-(n+1) d}
$$

if $h$ is stable of level $n$.

6.1.1. Proposition. Let $h$ and $h^{\prime}$ be in $\operatorname{Def}_{k}(\mathcal{L}(X))_{s t}$. Then

(1) $\tilde{\nu}\left(h \cup h^{\prime}\right)=\tilde{\nu}(h)+\tilde{\nu}\left(h^{\prime}\right)-\tilde{\nu}\left(h \cap h^{\prime}\right)$.

(2) If $h \approx h^{\prime}$, then $\tilde{\nu}(h)=\tilde{\nu}\left(h^{\prime}\right)$.

Proof. The first assertion follows directly from Proposition 3.4.4. The second assertion is a consequence of Proposition 4.4.2, together with the fact that $\pi_{n}(h) \approx$ $\pi_{n}\left(h^{\prime}\right)$ if $h \approx h^{\prime}$.

Now let $h$ be a stable definable subassignment of $h_{\mathcal{L}(X)}$ and consider a definable partition of $h$ with parameters in $\mathbf{Z}$ such that each $h_{n}$ is stable. Then, by Lemma 5.8.1. $\tilde{\nu}\left(h_{n}\right)=0$ for $|n| \gg 0$ and the sum $\sum_{n \in \mathbf{Z}} \mathbf{L}^{-n} \tilde{\nu}\left(h_{n}\right)$ is finite. We denote that sum by $\int \mathbf{L}^{-n} h_{n} d \tilde{\nu}$.

In general, for $h$ a definable subassignment of $h_{\mathcal{L}(X)}$ which is possibly not stable, one has to use a limit process to define a "motivic measure" of $h$. To achieve this aim, one needs the following lemma.

6.1.2. Lemma. Let $X$ be a variety over $k$ of dimension $d$ and let $h$ be a definable subassignment of $h_{\mathcal{L}(X)}$. Then there exist definable subassignments $k_{i}$, stable at level $n_{i}$, depending on the parameter $i \in \mathbf{N}$, and a closed subvariety $S$ of dimension $<d$ of $X$ such that

$$
h=\bigcup_{i} k_{i} \dot{\cup}\left(h \cap h_{\mathcal{L}(S)}\right), \quad \lim _{i \rightarrow \infty}\left(\operatorname{dim} \pi_{n_{i}}\left(k_{i}\right)-\left(n_{i}+1\right) d\right)=-\infty,
$$

with the definition of 4.5 , and such that the denominators of the elements $\chi_{c}\left(\pi_{n_{i}}\left(k_{i}\right)\right)$ in $K_{0}^{v}\left(\operatorname{Mot}_{k, \overline{\mathbf{Q}}}\right)_{\mathbf{Q}}, i \in \mathbf{N}$, are bounded. Furthermore, if $h_{n}, n \in \mathbf{Z}$, is a definable partition of $h$, it is possible to choose the $k_{i}$ 's in such a way that, for every $i, k_{i}$ is contained in some $h_{n}$.

Proof. One reduces first to the case when $X$ is affine irreducible with a closed immersion $X \rightarrow \mathbf{A}_{k}^{m}$, and then, by Proposition 5.5.1, we may assume $h$ is associated to a special formula $\varphi$ on $k[[t]]^{m}$ which is obtained by repeated application of conjunction and negation from formulas of the form (5.3.1), (5.3.2) and (5.3.3). Choose a nonzero regular function $g$ on $X$ which vanishes on the singular locus of $X$ and let $f$ denote the product of $g$ and all the polynomials $f_{i}$, assumed to be nonzero, occurring in (5.3.1), (5.3.2) and (5.3.3). Now set $\psi_{i}=\varphi \wedge \bigcap\left(\operatorname{ord}_{t} f=i\right)$ and define $S$ as the locus of $f=0$. The definable subassignments $k_{i}$ defined by $\psi_{i}$ are stable by Remark 5.8.2 and the statement on dimension follows from Lemma 4.4 of [12]. 
We still have to check that the denominators of $\chi_{c}\left(\pi_{n_{i}}\left(k_{i}\right)\right)$ in $K_{0}^{v}\left(\operatorname{Mot}_{k, \overline{\mathbf{Q}}}\right)_{\mathbf{Q}}$ are bounded. For that it is enough to know that one can uniformly bound the degree of the coverings in the Galois stratifications associated by quantifier elimination (Corollary [2.3.2) to $\pi_{n_{i}}\left(k_{i}\right)$. But consider the Galois stratifications associated by Corollary 2.3.2 to the formulas $\vartheta$ occurring in (5.3.3) and let $d$ be the maximum of the degrees of the coverings appearing in these Galois stratifications. The Galois stratifications associated to $\pi_{n_{i}}\left(\psi_{i}\right)$ may be expressed in terms of the former ones, and the integer $d$ is still a bound for the degrees of the coverings. For definable partitions, the construction of the $\psi_{i}$ 's is done in exactly the same way.

6.1.3. Definition-Theorem. There exists a unique mapping

$$
\nu: \operatorname{Def}_{k}(\mathcal{L}(X)) \longrightarrow \widehat{K}_{0}^{v}\left(\operatorname{Mot}_{k, \overline{\mathbf{Q}}}\right)_{\mathbf{Q}}
$$

satisfying the following properties.

(1) If $h$ is a stable definable subassignment of $h_{\mathcal{L}(X)}$, then $\nu(h)$ is equal to the image of $\tilde{\nu}(h)$ in $\widehat{K}_{0}^{v}\left(\operatorname{Mot}_{k, \overline{\mathbf{Q}}}\right)_{\mathbf{Q}}$.

(2) If $h$ and $h^{\prime}$ are definable subassignments of $h_{\mathcal{L}(X)}$, then

$$
\nu\left(h \cup h^{\prime}\right)=\nu(h)+\nu\left(h^{\prime}\right)-\nu\left(h \cap h^{\prime}\right) .
$$

(3) If $h \approx h^{\prime}$, then $\nu(h)=\nu\left(h^{\prime}\right)$.

(4) Let $h$ be a definable subassignment of $h_{\mathcal{L}(X)}$. If there exists a subvariety $S$ of $X$ with $\operatorname{dim} S \leq d-1$ such that $h \subset h_{\mathcal{L}(S)}$, then $\nu(h)=0$.

(5) Let $h_{n}$ be a definable partition of a definable subassignment $h$ with parameter $n \in \mathbf{N}$. Then the series $\sum_{n \in \mathbf{N}} \nu\left(h_{n}\right)$ is convergent in $\widehat{K}_{0}^{v}\left(\operatorname{Mot}_{k, \overline{\mathbf{Q}}}\right)_{\mathbf{Q}}$ and

$$
\nu(h)=\sum_{n \in \mathbf{N}} \nu\left(h_{n}\right) .
$$

(6) Let $h$ and $h^{\prime}$ be definable subassignments of $h_{\mathcal{L}(X)}$. Assume $h \subset h^{\prime}$. If $\nu\left(h^{\prime}\right)$ belongs to $F^{e} \widehat{K}_{0}^{v}\left(\operatorname{Mot}_{k, \overline{\mathbf{Q}}}\right)_{\mathbf{Q}}$, then $\nu(h)$ also belongs to $F^{e} \widehat{K}_{0}^{v}\left(\operatorname{Mot}_{k, \overline{\mathbf{Q}}}\right)_{\mathbf{Q}}$.

We call $\nu(h)$ the arithmetic motivic volume of $h$.

Proof. The proof is just the same as the proof of Definition-Proposition 3.2 in [12], if one replaces Lemma 2.4 and Lemma 3.1 of loc. cit. by Lemma 5.8.1 and Lemma 6.1 .2 .

Let $h_{n}$ be a definable partition of a definable subassignment $h$ with parameter $n \in \mathbf{Z}$. We say that $\mathbf{L}^{-n} h_{n}$ is integrable if the series

$$
\int \mathbf{L}^{-n} h_{n} d \nu:=\sum_{n \in \mathbf{Z}} \mathbf{L}^{-n} \nu\left(h_{n}\right)
$$

converges in $\widehat{K}_{0}^{v}\left(\operatorname{Mot}_{k, \overline{\mathbf{Q}}}\right)_{\mathbf{Q}}$. It follows from 6.1.3 (6) that if $h_{n}$ is a definable partition of a formula $h$ with parameter $n \in \mathbf{N}$ (or in $\mathbf{N} \cup\{\infty\}$ with the convention $\left.\mathbf{L}^{-\infty} \nu\left(h_{\infty}\right)=0\right)$, then $\mathbf{L}^{-n} h_{n}$ is integrable.

The following result is the analogue of Theorem 7.1 of [12] in the present context.

6.1.4. Theorem. Let $X$ be a variety over $k$ of dimension $d$. Let $h$ be a definable subassignment of $h_{\mathcal{L}(X)}$. Then

$$
\lim _{n \rightarrow \infty} \chi_{c}\left(\pi_{n}(h)\right) \mathbf{L}^{-(n+1) d}=\nu(h)
$$

in $\widehat{K}_{0}^{v}\left(\operatorname{Mot}_{k, \overline{\mathbf{Q}}}\right)_{\mathbf{Q}}$. 
Proof. Again the proof is essentially the same as the proof of Theorem 7.1 in [12], if one replaces Lemma 2.4 and Lemma 3.1 of loc. cit. by Lemma 5.8.1 and Lemma 6.1 .2

6.2. Change of variable formula. Let $X$ be an algebraic variety over $k$ of dimension $d$, and let $\mathcal{I}$ be a coherent sheaf of ideals on $X$. We denote by $\operatorname{ord}_{t} \mathcal{I}$ the function $\operatorname{ord}_{t} \mathcal{I}: \mathcal{L}(X) \rightarrow \mathbf{N} \cup\{\infty\}$ given by $\varphi \mapsto \min _{g} \operatorname{ord}_{t} g(\varphi)$, where the minimum is taken over all $g$ in the stalk $\mathcal{I}_{\pi_{0}(\varphi)}$ of $\mathcal{I}$ at $\pi_{0}(\varphi)$. Let $\Omega_{X}^{1}$ be the sheaf of differentials on $X$ and let $\Omega_{X}^{d}$ be the $d$-th exterior power of $\Omega_{X}^{1}$. If $X$ is smooth and $\mathcal{F}$ is a coherent sheaf on $X$ together with a natural morphism $\iota: \mathcal{F} \rightarrow \Omega_{X}^{d}$, we denote by $\mathcal{I}(\mathcal{F})$ the sheaf of ideals on $X$ which is locally generated by functions $\iota(\omega) / d x$ with $\omega$ a local section of $\mathcal{F}$ and $d x$ a local generator of $\Omega_{X}^{d}$. For $n \in \mathbf{N} \cup\{\infty\}$, we shall denote by $\operatorname{ord}_{t} \mathcal{F}=n$ the definable subassignment

$$
K \longmapsto\left\{x \in X(K[[t]]) \mid \operatorname{ord}_{t} \mathcal{I}(\mathcal{F})(x)=n\right\} .
$$

The subassignments $\operatorname{ord}_{t} \mathcal{F}=n, n \in \mathbf{N} \cup\{\infty\}$, form a definable partition of the functor $h_{\mathcal{L}(X)}$.

6.2.1. Theorem. Let $X$ and $Y$ be irreducible algebraic varieties over $k$ of dimension $m$. Assume $Y$ is smooth. Let $p: Y \rightarrow X$ be a proper birational morphism. Let $h_{n}$ be a definable partition of a definable subassignment $h$ of $h_{\mathcal{L}(X)}$ with parameter $n \in \mathbf{N}$. Consider the definable subassignment $k_{n}$ of $h_{\mathcal{L}(Y)}$ defined by

$$
k_{n}:=\bigcup_{i+j=n} p^{-1}\left(h_{i}\right) \cap\left(\operatorname{ord}_{t} p^{*} \Omega_{X}^{d}=j\right) .
$$

The $k_{n}$ 's, $n \in \mathbf{N} \cup\{\infty\}$, form a definable partition of $p^{-1}(h)$ and

$$
\int \mathbf{L}^{-n} h_{n} d \nu_{X}=\int \mathbf{L}^{-n} k_{n} d \nu_{Y}
$$

Furthermore, if $h \cap h_{\mathcal{L}(p(E))}=\emptyset$, with $E$ the exceptional locus of $p$, and $h$ and the $h_{n}$ 's are weakly stable (hence stable by Remark 5.8.2), then $p^{-1}(h)$ and the $k_{n}$ 's are stable and the above formula still holds when $\nu$ is replaced by $\tilde{\nu}$.

Proof. The proof follows from Lemma 3.4 in 12 similarly as the proof of Lemma 3.3 in 12 .

\section{RAtionality RESUlts}

In this section we prove analogues of rationality results in [12].

7.1. We consider the ring $\widehat{K}_{0}^{v}\left(\operatorname{Mot}_{k, \overline{\mathbf{Q}}}\right)_{\mathbf{Q}}[[T]]$ of power series in the variable $T=$ $\left(T_{1}, \ldots, T_{r}\right)$ with coefficients in $\widehat{K}_{0}^{v}\left(\operatorname{Mot}_{k, \overline{\mathbf{Q}}}\right)_{\mathbf{Q}}$. We denote by

$$
\bar{K}_{0}^{v}\left(\operatorname{Mot}_{k, \overline{\mathbf{Q}}}\right)_{\mathrm{loc}, \mathbf{Q}}[[T]]_{\mathrm{rat}}
$$

the subring of $\widehat{K}_{0}^{v}\left(\operatorname{Mot}_{k, \overline{\mathbf{Q}}}\right)_{\mathbf{Q}}[[T]]$ generated by $\bar{K}_{0}^{v}\left(\operatorname{Mot}_{k, \overline{\mathbf{Q}}}\right)_{\text {loc, } \mathbf{Q}}[T],\left(\mathbf{L}^{i}-1\right)^{-1}$ and $\left(1-\mathbf{L}^{-a} T^{b}\right)^{-1}$, with $i \in \mathbf{N} \backslash\{0\}, a \in \mathbf{N}$, and $b \in \mathbf{N}^{r} \backslash\{0\}$. 
7.1.1. Theorem. Let $X$ be an algebraic variety over $k$ of dimension $d$. Let $h_{n}$ be a definable subassignment of $h_{\mathcal{L}(X)}$ depending on the parameter $n \in \mathbf{N}^{r}$, and let $h_{n, i}$ be a definable partition of the $h_{n}$ 's depending on the parameter $(n, i) \in \mathbf{N}^{r} \times \mathbf{N}$. Then the power series

$$
\sum_{n \in \mathbf{N}^{r}} \int \mathbf{L}^{-i} h_{n, i} d \nu T^{n}
$$

in the variable $T=\left(T_{1}, \ldots, T_{r}\right)$ belongs to $\bar{K}_{0}^{v}\left(\operatorname{Mot}_{k, \overline{\mathbf{Q}}}\right)_{\mathrm{loc}, \mathbf{Q}}[[T]]_{\mathrm{rat}}$.

7.1.2. Corollary. For every definable subassignment $h$ of $h_{\mathcal{L}(X)}$, the measure $\nu(h)$ belongs to the subring $\bar{K}_{0}^{v}\left(\operatorname{Mot}_{k, \overline{\mathbf{Q}}}\right)_{\mathrm{loc}, \mathbf{Q}}\left[\left(\left(\mathbf{L}^{i}-1\right)^{-1}\right)_{i \geq 1}\right]$ of $\widehat{K}_{0}^{v}\left(\operatorname{Mot}_{k, \overline{\mathbf{Q}}}\right)_{\mathbf{Q}}$.

7.2. Let us denote by $K_{0}^{v}\left(\operatorname{Mot}_{k, \overline{\mathbf{Q}}}\right)_{\text {loc, } \mathbf{Q}}[[T]]_{\mathrm{rat}}$ the subring of $K_{0}^{v}\left(\operatorname{Mot}_{k, \overline{\mathbf{Q}}}\right)_{\mathrm{loc}, \mathbf{Q}}[[T]]$ generated by $K_{0}^{v}\left(\operatorname{Mot}_{k, \overline{\mathbf{Q}}}\right)_{\text {loc, } \mathbf{Q}}[T]$ and the series $\left(1-\mathbf{L}^{-a} T^{b}\right)^{-1}$, with $a \in \mathbf{N}$ and $b \in \mathbf{N}^{r} \backslash\{0\}$.

7.2.1. Theorem. Let $X$ be an algebraic variety over $k$ of dimension $d$. Let $h_{n}$ be a definable subassignment of $h_{\mathcal{L}(X)}$ depending on the parameter $n \in \mathbf{N}^{r}$, and let $h_{n, i}$ be a definable partition of the $h_{n}$ 's depending on the parameter $(n, i) \in \mathbf{N}^{r} \times \mathbf{N}$. Assume $h_{n} \cap h_{\mathcal{L}\left(X_{0}\right)}=\emptyset$, for every $n \in \mathbf{N}^{r}$, with $X_{0}$ the union of the singular locus of $X$ and its irreducible components of dimension $<d$ and that the subassignments $h_{n}$ and $h_{n, i}$ are all weakly stable (hence stable by Remark 5.8.2). Then the power series

$$
\sum_{n \in \mathbf{N}^{r}} \int \mathbf{L}^{-i} h_{n, i} d \tilde{\nu} T^{n}
$$

in the variable $T=\left(T_{1}, \ldots, T_{r}\right)$ belongs to $K_{0}^{v}\left(\operatorname{Mot}_{k, \overline{\mathbf{Q}}}\right)_{\mathrm{loc}, \mathbf{Q}}[[T]]_{\mathrm{rat}}$.

7.3. For the proof of Theorem 7.2.1 one needs the following technical lemma on bounded representations.

We shall say that a definable subassignment $h_{\ell}$, depending on parameters $\ell \in \mathbf{N}^{n}$, of $h_{\mathcal{L}(X)}$ has a bounded representation if there exists a covering of $X$ by affine Zariski open sets $X_{i}$ embedded in $\mathbf{A}_{k}^{m_{i}}$ such that $h_{\ell} \cap h_{\mathcal{L}\left(X_{i}\right)}$ is associated to a special formula on $k[[t]]^{m_{i}}$ which is obtained by repeated application of conjunction and negation from formulas of the form (5.3.1), (5.3.2) and (5.3.3) with $\operatorname{ord}_{t} f_{i}$ bounded on $h_{\ell} \cap h_{\mathcal{L}\left(X_{i}\right)}$ for each fixed $\ell$. Here by "bounded" we mean there exists a number $\kappa$ depending on $\ell$ such that $\operatorname{ord}_{t} f_{i} \leq \kappa$, for every $\gamma$ in $h_{\ell} \cap h_{\mathcal{L}\left(X_{i}\right)}(L)$ and every $L$ in Field $_{k}$.

Clearly, if the family $h_{\ell}$ has a bounded representation, then each $h_{\ell}$ is weakly stable.

7.3.1. Lemma. Let $X$ be a quasi-projective algebraic variety over $k$ and let $h_{\ell}$, $\ell \in \mathbf{N}^{n}$, be a definable subassignment of $h_{\mathcal{L}(X)}$ depending on parameters $\ell \in \mathbf{N}^{n}$. Assume that $h_{\ell}$ is weakly stable for each $\ell$. Then the family $h_{\ell}$ is a finite boolean combination of definable subassignments of $h_{\mathcal{L}(X)}$ depending on parameters which have bounded representations.

Proof. The proof of Lemma 2.8 of [12] may be directly adapted to carry over to the present situation. 
7.4. The proofs of Theorems 7.1.1 and 7.2.1 are quite similar to the ones of Theorem 5.1 and Theorem 5.1' in [12]. We give details which will be used in section 8

Proof of Theorems 7.1.1 and 7.2.1. Let us first prove Theorem 7.2.1. By Theorem 6.2 .1 one may use a resolution of singularities and assume that $X$ is smooth. Also we may assume $X$ is affine. By Pas's Theorem, Corollary [5.1.2 and Lemma 5.8.1 there exists a Presburger function $\theta: \mathbf{N}^{r} \rightarrow \mathbf{N}$ such that the series (7.2.2) is equal to

$$
\sum_{i \leq \theta(n), n \in \mathbf{N}^{r}} \tilde{\nu}\left(h_{n, i}\right) \mathbf{L}^{-i} T^{n}
$$

By Lemma 7.3.1 one reduces as in 12] to the case where the family $\left(h_{n, i}\right)_{(n, i)}$ has bounded representation. Furthermore, one may assume $X=X_{i}$ in the bounded representation of the family $\left(h_{n, i}\right)_{(n, i)}$ by special formulas obtained by repeated application of conjunction and negation from formulas of the form (5.3.1), (5.3.2) and (5.3.3). We denote by $F$ the product of all the polynomials $f_{i}$, assumed to be nonzero, occurring in these formulas of the form (5.3.1), (5.3.2) and (5.3.3) and we consider an embedded resolution of singularities $\gamma: Y \rightarrow X$ of the locus of $F=0$ in $X$, with exceptional locus contained in $\gamma^{-1}\left(F^{-1}(0)\right)$. The variety $Y$ admits a covering by affine open subsets $U$ on which there exist regular functions $z_{1}, \ldots, z_{d}$ inducing an étale map $U \rightarrow \mathbf{A}_{k}^{d}$ such that on $U$ each $f_{i} \circ \gamma$ is a monomial in $z_{1}, \ldots, z_{d}$ multiplied by a regular function with no zeros on $U$. One may assume furthermore that the variables $z_{i}$ appearing in at least one of these monomials are exactly $z_{1}, z_{2}, \ldots, z_{d_{0}}$.

Now for $w$ a definable subassignment of $h_{\left(\mathbf{A}_{k}^{1} \backslash\{0\}\right)^{d_{0}} \times U}$ and $\ell_{1}, \ldots, \ell_{d_{0}}$ in $\mathbf{N}$, we denote by $h_{w, \ell_{1}, \ldots, \ell_{d_{0}}}$ the definable subassignment of $h_{\mathcal{L}(U)}$ defined by

$$
\begin{aligned}
K \longmapsto\left\{x \in \mathcal{L}(U)(K) \mid \operatorname{ord}_{t} z_{i}(x)=\ell_{i}, 1 \leq i \leq d_{0},\right. \\
\left.\quad \text { and }\left(\left(\overline{\operatorname{ac}}\left(z_{i}(x)\right)\right), \pi_{0}(x)\right) \in w(K)\right\} .
\end{aligned}
$$

It now follows from Theorem 6.2.1 and the fact that $\operatorname{ord}_{t} F$ is bounded on $h_{n, i}$ that, uniformly in $n, i, \tilde{\nu}\left(h_{n, i}\right)$ is a finite $\mathbf{Z}$-linear combination of terms of the form

$$
\sum_{\substack{\ell_{1}, \ldots, \ell_{d_{0}} \in \mathbf{N} \\ \theta\left(\ell_{1}, \ldots, \ell_{d_{0}}, n, i\right)}} \mathbf{L}^{-\beta\left(\ell_{1}, \ldots, \ell_{d_{0}}\right)} \tilde{\nu}\left(h_{w, \ell_{1}, \ldots, \ell_{d_{0}}}\right),
$$

where $\theta\left(\ell_{1}, \ldots, \ell_{d_{0}}, n, i\right)$ is a condition defining a Presburger subset of $\mathbf{Z}^{d_{0}+r+1}$, $\beta$ is a linear form with coefficients in $\mathbf{N}$, and $w$ is a definable subassignment of $h_{\left(\mathbf{A}_{k}^{1} \backslash\{0\}\right)^{d_{0}} \times U}$, for $U$ as above. Since $\operatorname{ord}_{t} F$ is bounded on $h_{n, i}$, the sum in (7.4.5) is finite.

Let us denote by $\bar{w}$ the definable subassignment of $h_{\left(\mathbf{A}_{k}^{1} \backslash\{0\}\right)^{d_{0} \times U}}$ defined as $\bar{w}=w \cap h_{U^{\prime}}$ with $U^{\prime}$ the subvariety of $\left(\mathbf{A}_{k}^{1} \backslash\{0\}\right)^{d_{0}} \times U$ defined as the locus of points $\left(w_{1}, \ldots, w_{d_{0}}, y\right)$ in $\left(\mathbf{A}_{k}^{1} \backslash\{0\}\right)^{d_{0}} \times U$ such that $z_{i}(y)=0$ when $\ell_{i}>0$ and $z_{i}(y)=w_{i}$ when $\ell_{i}=0$. But, by Lemma 4.1 of [12], with $n=e=0$, we have

$$
\tilde{\nu}\left(h_{w, \ell_{1}, \ldots, \ell_{d_{0}}}\right)=\chi_{c}(\bar{w}) \mathbf{L}^{-\left(\sum_{i=1}^{d_{0}} \ell_{i}\right)-d},
$$


hence we can rewrite, uniformly in $n, i, \tilde{\nu}\left(h_{n, i}\right)$ as a finite $\mathbf{Z}$-linear combination of terms of the form

$$
\mathbf{L}^{-d} \sum_{\substack{\ell_{1}, \ldots, \ell_{d_{0}} \in \mathbf{N} \\ \theta\left(\ell_{1}, \ldots, \ell_{d_{0}}, n, i\right)}} \mathbf{L}^{-\beta\left(\ell_{1}, \ldots, \ell_{d_{0}}\right)} \chi_{c}(\bar{w}),
$$

with $\theta, \beta$ and $\bar{w}$ as above.

As in [12], one may now conclude the proof by using Lemma 5.2 and Lemma 5.3 of [12].

The proof of Theorem 7.1.1 is similar except for the fact that we have to replace $\tilde{\nu}$ by $\nu$ and the finite sums are replaced by infinite sums which converge in $\widehat{K}_{0}^{v}\left(\operatorname{Mot}_{k, \overline{\mathbf{Q}}}\right)_{\mathbf{Q}}[[T]]$. In particular, $\nu\left(h_{n, i}\right)$ is still a finite $\mathbf{Z}$-linear combination of terms of the form (7.4.7), but the number of terms in the series (7.4.7) may now be infinite.

\section{Arithmetic motivic integration SpeCializes to $p$-ADiC integration}

8.1. Again let $U=\operatorname{Spec} R$ denote an affine scheme of finite type over $\mathbf{Z}$, which is assumed to be integral and normal, with fraction field $k$ of characteristic zero.

\subsubsection{Lemma. The morphism}

$$
\mathrm{Ét}_{\ell}: K_{0}^{v}\left(\operatorname{Mot}_{k, \overline{\mathbf{Q}}}\right)_{\mathrm{loc}, \mathbf{Q}} \longrightarrow K_{0}\left(G_{k}, \overline{\mathbf{Q}}_{\ell}\right) \otimes \mathbf{Q}
$$

induced by étale $\ell$-adic realization factorizes through a morphism

$$
\mathrm{É}_{\ell}: \bar{K}_{0}^{v}\left(\operatorname{Mot}_{k, \overline{\mathbf{Q}}}\right)_{\mathrm{loc}, \mathbf{Q}} \longrightarrow K_{0}\left(G_{k}, \overline{\mathbf{Q}}_{\ell}\right) \otimes \mathbf{Q} \text {. }
$$

In particular, for $x$ in $U$, the morphism

$$
\operatorname{Tr}_{\operatorname{Frob}}: K_{0}^{v}\left(\operatorname{Mot}_{k, \overline{\mathbf{Q}}}\right)_{\mathrm{loc}, \mathbf{Q}} \longrightarrow \overline{\mathbf{Q}}_{\ell}
$$

factorizes through a morphism

$$
\operatorname{Tr} \operatorname{Frob}_{x}: \bar{K}_{0}^{v}\left(\operatorname{Mot}_{k, \overline{\mathbf{Q}}}\right)_{\text {loc }, \mathbf{Q}} \longrightarrow \overline{\mathbf{Q}}_{\ell}
$$

Proof. For $\alpha$ in $K_{0}^{v}\left(\operatorname{Mot}_{k, \overline{\mathbf{Q}}}\right)_{\text {loc }}$, denote by $P_{\alpha, x}(T)$ the "characteristic polynomial" $\operatorname{det}\left(1-\operatorname{Frob}_{x} T\right)$ of $\operatorname{Frob}_{x}$ on $\operatorname{É}_{\ell}(\alpha)$ (since we are dealing with virtual representations, $P_{\alpha, x}(T)$ is a rational function). Assume $\alpha$ is in $\bigcap_{m} F^{m} K_{0}^{v}\left(\operatorname{Mot}_{k, \overline{\mathbf{Q}}}\right)_{\text {loc }}$ for every $m$ in $\mathbf{Z}$. By the part of the Weil conjectures proven by Deligne [6], we then have $P_{\alpha, x}(T)=1$ for $x$ in a set of Dirichlet density 1 . Since a virtual $\ell$-adic representation of $G_{k}$ is determined by the corresponding characteristic polynomials, we deduce from Chebotarev's Theorem that $\operatorname{É}_{\ell}(\alpha)=1$. The result follows.

We may extend the morphism

$$
\operatorname{Tr} \operatorname{Frob}_{x}: \bar{K}_{0}^{v}\left(\operatorname{Mot}_{k, \overline{\mathbf{Q}}}\right)_{\text {loc }, \mathbf{Q}} \longrightarrow \overline{\mathbf{Q}}_{\ell}
$$

to a morphism

$$
\operatorname{Tr} \operatorname{Frob}_{x}: \bar{K}_{0}^{v}\left(\operatorname{Mot}_{k, \overline{\mathbf{Q}}}\right)_{\mathrm{loc}, \mathbf{Q}}\left[\left(\left(\mathbf{L}^{i}-1\right)^{-1}\right)_{i \geq 1}\right] \longrightarrow \overline{\mathbf{Q}}_{\ell},
$$

by sending $\left(\mathbf{L}^{i}-1\right)^{-1}$ to $\left(q_{x}^{i}-1\right)^{-1}$, with $q_{x}$ the cardinality of $\mathbf{F}_{x}$.

8.1.2. Remark. Let $\alpha$ be in one of the rings $K_{0}^{v}\left(\operatorname{Mot}_{k, \overline{\mathbf{Q}}}\right)_{\text {loc, }, \mathbf{Q}}, \bar{K}_{0}^{v}\left(\operatorname{Mot}_{k, \overline{\mathbf{Q}}}\right)_{\text {loc, } \mathbf{Q}}$, or $\bar{K}_{0}^{v}\left(\operatorname{Mot}_{k, \overline{\mathbf{Q}}}\right)_{\text {loc, } \mathbf{Q}}\left[\left(\left(\mathbf{L}^{i}-1\right)^{-1}\right)_{i \geq 1}\right]$. For $x$ in a dense open subset of $U, \operatorname{Tr} \operatorname{Frob}_{x}(\alpha)$ is in fact a rational number, since it is a $\mathbf{Q}$-linear combination of numbers of rational points of varieties over the residual field $\mathbf{F}_{x}$. In particular, it follows from Corollary 
7.1.2 that, for every definable subassignment $h$ of $h_{\mathcal{L}(X)}, \operatorname{Tr} \operatorname{Frob}_{x}(\nu(h))$ is a rational number for $x$ in a dense open subset of $U$.

Similarly, the morphism $\operatorname{Tr} \operatorname{Frob}_{x}$ may be naturally extended to a morphism

$$
\operatorname{Tr} \operatorname{Frob}_{x}: \bar{K}_{0}^{v}\left(\operatorname{Mot}_{\mathbf{Q}, \overline{\mathbf{Q}}}\right)_{\text {loc }, \mathbf{Q}}[[T]]_{\mathrm{rat}} \longrightarrow \overline{\mathbf{Q}}_{\ell}(T) \text {. }
$$

8.2. Let $K$ be the field of fractions of a complete discrete valuation ring $\mathcal{O}_{K}$ with finite residue field $\mathbf{F}_{K}$. We set $q=\operatorname{card} \mathbf{F}_{K}$ and we fix a uniformizing parameter $\pi$. We normalize the valuation ord on $K$ by $\operatorname{ord}(\pi)=1$, and we define, for $x \neq 0$ in $K$, $\overline{\operatorname{ac}} x=\pi^{-\operatorname{ord}(x)} x \bmod \pi$ and $|x|=q^{-\operatorname{ord}(x)}$, and $\overline{\operatorname{ac}} 0=|0|=0$. We obtain in this way a structure for the language $\mathcal{L}_{\text {Pas }}$. By a definable subset of $\mathcal{O}_{K}^{n}=\mathbf{A}^{n}\left(\mathcal{O}_{K}\right)$, we shall mean a subset defined by an $\mathcal{L}_{\text {Pas }}$-formula with coefficients in $K$ in the valued field sort and coefficients in $\mathbf{F}_{K}$ in the residue field sort, $n$ free variables running over $K$ and no other free variables. More generally, for $\mathcal{X}$ an algebraic variety over $\mathcal{O}_{K}$, one defines definable subsets of $\mathcal{X}\left(\mathcal{O}_{K}\right)$ by taking covers by affine open subvarieties. Similarly, one defines definable subsets of $\mathcal{X}\left(\mathcal{O}_{K}\right)$ depending on parameters in $\mathbf{Z}^{r}, \mathbf{N}^{r}$, or $\mathbf{N} \cup\{\infty\}$.

Let $d$ be the Krull dimension of $\mathcal{X} \otimes K$. There is a natural $d$-dimensional measure on $\mathcal{X}\left(\mathcal{O}_{K}\right)$ (cf. 30, 25), which we shall denote by $\nu_{K}$, for which all definable subsets of $\mathcal{X}\left(\mathcal{O}_{K}\right)$ are measurable, when $K$ is of characteristic zero (cf. [8, [9], 31]). This measure is defined by requiring that the fibers of the reduction map modulo $\pi$ have measure $q^{-d}$.

8.3. We assume now $k$ is a finite extension of $\mathbf{Q}$ with ring of integers $\mathcal{O}$ and $R=\mathcal{O}\left[\frac{1}{N}\right]$, for some nonzero integer $N$ which is a multiple of the discriminant of $k$. For $x$ a closed point of Spec $R$, we denote by $\mathcal{O}_{K_{x}}$ the completion of the localization of $R$ at $x$, by $K_{x}$ its field of fractions and by $\mathbf{F}_{x}$ the residue field at $x$.

Let $\varphi$ be a formula over $R[[t]]^{m}$. We denote by $Z\left(\varphi, \mathcal{O}_{K_{x}}\right)$ the subset of $\mathcal{O}_{K_{x}}^{m}$ defined by the formula $\varphi$. By Proposition 5.2.1 if $\varphi^{\prime}$ is another formula over $R[[t]]^{m}$ defining the same definable subassignment of $h_{\mathcal{L}\left(\mathbf{A}_{R}^{m}\right)}$ as $\varphi$, we have

$$
Z\left(\varphi, \mathcal{O}_{K_{x}}\right)=Z\left(\varphi^{\prime}, \mathcal{O}_{K_{x}}\right)
$$

for almost all $x$. Now let $\mathcal{X}$ be a variety over $R$ and let $h$ be a definable subassignment of $h_{\mathcal{L}(\mathcal{X})}$. Take a covering of $\mathcal{X}$ by affine open subvarieties $U_{i}$ together with formulas $\varphi_{i}$ defining $h$ on $U_{i}$. By the preceding observation, for almost all closed points $x$ of Spec $R$, the subsets $Z\left(\varphi_{i}, \mathcal{O}_{K_{x}}\right)$ of $U_{i}\left(\mathcal{O}_{K_{x}}\right)$ may be glued together to define a subset of $\mathcal{X}\left(\mathcal{O}_{K_{x}}\right)$, which we will denote by $h\left[\mathcal{O}_{K_{x}}\right]$. This subset $h\left[\mathcal{O}_{K_{x}}\right]$ depends on choices, but two different choices yield the same $h\left[\mathcal{O}_{K_{x}}\right]$ for almost all $x$.

As the following result shows, $p$-adic integration may be viewed as a specialization of arithmetic motivic integration.

8.3.1. Theorem. Let $k$ be a finite extension of $\mathbf{Q}$ with ring of integers $\mathcal{O}$ and $R=\mathcal{O}\left[\frac{1}{N}\right]$, for some nonzero integer $N$. Let $\mathcal{X}$ be a variety over $R$ and let $h$ be a definable subassignment of $h_{\mathcal{L}(\mathcal{X})}$. Then there exists a nonzero multiple $N^{\prime}$ of $N$, such that, for every closed point $x$ of $\operatorname{Spec} \mathcal{O}\left[\frac{1}{N^{\prime}}\right]$,

$$
\operatorname{Tr}_{\operatorname{Frob}}(\nu(h \otimes k))=\nu_{K_{x}}\left(h\left[\mathcal{O}_{K_{x}}\right]\right) .
$$

More generally, if $h_{n}$ is a definable subassignment of $h_{\mathcal{L}(\mathcal{X})}$ depending on the parameter $n \in \mathbf{N}^{r}$, and if $h_{n, i}$ is a definable partition of the $h_{n}$ 's depending on the 
parameter $(n, i) \in \mathbf{N}^{r} \times \mathbf{N}$, there exists a nonzero multiple $N^{\prime}$ of $N$, such that, for every closed point $x$ of Spec $\mathcal{O}\left[\frac{1}{N^{\prime}}\right]$, the series $\sum_{i \in \mathbf{N}} q_{x}^{-i} \nu_{K_{x}}\left(h_{n, i}\left[\mathcal{O}_{K_{x}}\right]\right)$ converges in $\mathbf{R}$ to a rational number, for every $n$ in $\mathbf{N}^{r}$, and the following equality of power series holds in $\mathbf{Q}(T)$ :

$$
\operatorname{Tr} \operatorname{Frob}_{x}\left(\sum_{n \in \mathbf{N}^{r}} \int \mathbf{L}^{-i}\left(h_{n, i} \otimes k\right) d \nu T^{n}\right)=\sum_{n \in \mathbf{N}^{r}} \sum_{i \in \mathbf{N}} q_{x}^{-i} \nu_{K_{x}}\left(h_{n, i}\left[\mathcal{O}_{K_{x}}\right]\right) T^{n} .
$$

Proof. We shall prove directly the more general second statement. The proof will proceed by comparison with the proof of Theorems 7.1.1 and 7.2.1. We shall make no notational difference here between $h$ and $h \otimes k$ and between $h_{n, i}$ and $h_{n, i} \otimes k$. We set $X=\mathcal{X} \otimes k$. In loc. cit. we first used a resolution of singularities of $X$ to reduce to the smooth case, and then considered an embedded resolution of a divisor $F=0$ on some affine open subsets of $X$. By Theorem 2.4 of [10], there exists a nonzero multiple $N^{\prime}$ of $N$ such that these resolutions extend over $\operatorname{Spec} \mathcal{O}\left[\frac{1}{N^{\prime}}\right]$ to resolutions with good reduction $\bmod P_{x}$, for every closed point $x$ in $\operatorname{Spec} \mathcal{O}\left[\frac{1}{N^{\prime}}\right]$, in the sense of [10]. Here $P_{x}$ denotes the maximal ideal at $x$. Now, by the local calculations of $p$-adic integrals on resolutions with good reduction in [10], we deduce that, for every closed point $x$ in $\operatorname{Spec} \mathcal{O}\left[\frac{1}{N^{\prime}}\right], \nu_{K_{x}}\left(h_{n, i}\left[\mathcal{O}_{K_{x}}\right]\right)$ is a finite $\mathbf{Z}$-linear combination of terms of the form

$$
q_{x}^{-d} \sum_{\substack{\ell_{1}, \ldots, \ell_{d_{0}} \in \mathbf{N} \\ \theta\left(\ell_{1}, \ldots, \ell_{d_{0}}, n, i\right)}} q_{x}^{-\beta\left(\ell_{1}, \ldots, \ell_{d_{0}}\right)} \operatorname{card} \bar{w}\left(\mathbf{F}_{x}\right),
$$

where $\theta, \beta$ and $\bar{w}$ are the same as in (7.4.7), and furthermore the coefficients of the terms 8.3 .2$)$ in $\nu_{K_{x}}\left(h_{n, i}\left[\mathcal{O}_{K_{x}}\right]\right)$ are the same as the coefficients of the terms (7.4.7) in $\nu\left(h_{n, i}\right)$. Indeed, we may assume $X$ is affine and, by Proposition 5.2.1 we may also assume, maybe after replacing $N$ by some nonzero multiple, that the subassignments $h_{n, i}$ are defined by repeated application of conjunction and negation from formulas of the form

$$
\begin{gathered}
\operatorname{ord} f_{1}\left(x_{1}, \ldots, x_{m}\right) \geq \operatorname{ord} f_{2}\left(x_{1}, \ldots, x_{m}\right)+L(n, i), \\
\quad \operatorname{ord} f_{1}\left(x_{1}, \ldots, x_{m}\right) \equiv L(n, i) \quad \bmod b
\end{gathered}
$$

and

$$
\vartheta\left(\overline{\operatorname{ac}}\left(f_{1}\left(x_{1}, \ldots, x_{m}\right)\right), \ldots, \overline{\operatorname{ac}}\left(f_{m^{\prime}}\left(x_{1}, \ldots, x_{m}\right)\right)\right),
$$

where $f_{i}$ are regular functions, $L$ is a polynomial with coefficients in $\mathbf{Z}$ and degree $\leq 1, b$ is in $\mathbf{Z}$, and $\vartheta$ is an $\mathbf{L}_{k}$-formula in $m^{\prime}$ free variables. Furthermore we may assume there exist regular functions $z_{1}, \ldots, z_{d}$ on $X$ inducing an étale map $X \rightarrow \mathbf{A}_{k}^{d}$ such that on $X$ each $f_{i}$ is a monomial in $z_{1}, \ldots, z_{d}$ multiplied by a regular function with no zeros on $X$, and that the variables $z_{i}$ appearing in at least one of these monomials are exactly $z_{1}, z_{2}, \ldots, z_{d_{0}}$.

With the notations from 7.4, we deduce from the local calculations in 7.4.7 that $\nu\left(h_{n, i}\right)$ is a $\mathbf{Z}$-linear combination of terms of the form

$$
\mathbf{L}^{-d} \sum_{\substack{\ell_{1}, \ldots, \ell_{d_{0}} \in \mathbf{N} \\ \theta\left(\ell_{1}, \ldots, \ell_{d_{0}}, n, i\right)}} \mathbf{L}^{-\beta\left(\ell_{1}, \ldots, \ell_{d_{0}}\right)} \chi_{c}(\bar{w})
$$


with $\theta\left(\ell_{1}, \ldots, \ell_{d_{0}}, n, i\right)$ a condition defining a Presburger subset of $\mathbf{Z}^{d_{0}+r+1}, \beta$ a linear form with coefficients in $\mathbf{N}$, and $\bar{w}$ a definable subassignment of $h_{\left(\mathbf{A}_{k}^{1} \backslash\{0\}\right)^{d_{0}} \times X}$. Here $\theta$ and $\beta$ depend only on the monomials appearing in the functions $f_{i}$, the coefficients of the polynomials $L$ and the integers $b$, and $\bar{w}$ depends furthermore on $\vartheta$. In the good reduction case, local calculation of $p$-adic integrals as performed in 10 just provides the same expression for $\nu_{K_{x}}\left(h_{n, i}\left[\mathcal{O}_{K_{x}}\right]\right)$ as a $\mathbf{Z}$-linear combination of terms of the form

$$
q^{-d} \sum_{\substack{\ell_{1}, \ldots, \ell_{d_{0}} \in \mathbf{N} \\ \theta\left(\ell_{1}, \ldots, \ell_{d_{0}}, n, i\right)}} q^{-\beta\left(\ell_{1}, \ldots, \ell_{d_{0}}\right)} \operatorname{card} \bar{w}\left(\mathbf{F}_{x}\right),
$$

with the same coefficients in $\mathbf{Z}$ as for $\nu\left(h_{n, i}\right)$, and for each term the same functions $\theta$ and $\beta$ and the same definable subassignment $\bar{w}$ as in (8.3.6). The result now follows, since, by Proposition 4.3.1,

$$
\operatorname{Tr} \operatorname{Frob}_{x}\left(\chi_{c}(\bar{w})\right)=\operatorname{card} \bar{w}\left(\mathbf{F}_{x}\right),
$$

for every closed point $x$ in Spec $\mathcal{O}\left[\frac{1}{N^{\prime \prime}}\right]$, for a suitable nonzero multiple $N^{\prime \prime}$ of $N^{\prime}$.

The following variant of Theorem 8.3.1 is proved in the same way.

8.3.2. Theorem. Let $k$ be a field of characteristic zero which is the field of fractions of a normal domain $R$ of finite type over $\mathbf{Z}$. Let $\mathcal{X}$ be a variety over $R$ and let $h$ be a definable subassignment of $h_{\mathcal{L}(\mathcal{X})}$. Then there exists a nonzero element $f$ of $R$, such that, for every closed point $x$ of $\operatorname{Spec} R_{f}, h\left(\mathbf{F}_{x}\right)$ is $\nu_{\mathbf{F}_{x}}[[t]]-$ measurable and

$$
\operatorname{Tr} \operatorname{Frob}_{x}(\nu(h \otimes k))=\nu_{\mathbf{F}_{x}[[t]]}\left(h\left(\mathbf{F}_{x}\right)\right) .
$$

More generally, if $h_{n}$ is a definable subassignment of $h_{\mathcal{L}(\mathcal{X})}$ depending on the parameter $n \in \mathbf{N}^{r}$, and if $h_{n, i}$ is a definable partition of the $h_{n}$ 's depending on the parameter $(n, i) \in \mathbf{N}^{r} \times \mathbf{N}$, then there exists a nonzero element $f$ of $R$, such that,

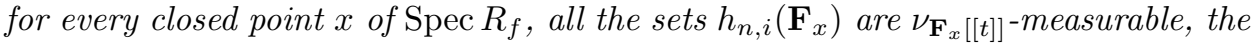
series $\sum_{i \in \mathbf{N}} q_{x}^{-i} \nu_{\left.\left.\mathbf{F}_{x}[t]\right]\right]}\left(h_{n, i}\left(\mathbf{F}_{x}\right)\right)$ converges in $\mathbf{R}$ to a rational number, for every $n$ in $\mathbf{N}^{r}$, and the following equality of power series holds in $\mathbf{Q}(T)$ :

$$
\operatorname{Tr} \operatorname{Frob}_{x}\left(\sum_{n \in \mathbf{N}^{r}} \int \mathbf{L}^{-i}\left(h_{n, i} \otimes k\right) d \nu T^{n}\right)=\sum_{n \in \mathbf{N}^{r}} \sum_{i \in \mathbf{N}} q_{x}^{-i} \nu_{\mathbf{F}_{x}[[t]]}\left(h_{n, i}\left(\mathbf{F}_{x}\right)\right) T^{n} .
$$

8.4. Again, let $k$ be a finite extension of $\mathbf{Q}$ with ring of integers $\mathcal{O}$. Consider a polynomial $f(x) \in k\left[x_{1}, \ldots, x_{m}\right]$ and let $\varphi$ be an $\mathcal{L}_{\text {Pas }}$-formula with coefficients in $k$ in the valued field sort and in the residue field sort, free variables $x_{1}, \ldots, x_{m}$ running over the valued field sort and no other free variables. Let $x$ be a closed point of $\operatorname{Spec} \mathcal{O}$ not dividing the discriminant of $k$ and set

$$
W_{x}:=\left\{y \in \mathcal{O}_{K_{x}}^{m} \mid \varphi(y) \text { holds in } K_{x}\right\} .
$$

We consider the $p$-adic integral

$$
I_{\varphi, f}(s, x):=\int_{W_{x}}|f(y)|_{x}^{s}|d y|_{x}
$$

where ||$_{x}$ and $|d y|_{x}$ denote the $p$-adic norm on $K_{x}$ and volume form on $K_{x}^{m}$, respectively. By a result of Denef [7], $I_{\varphi, f}(s, x)$ is a rational function of $q_{x}^{-s}$. Macintyre 
24], Pas [26] and Denef [10] proved that the degrees of the numerator and denominator of this rational function are bounded independently of $x$.

8.5. We will now show that a much stronger uniformity statement holds: the integrals $I_{\varphi, f}(s, x)$ may be interpolated in a canonical way by a "motivic rational function".

Let $k$ be a field of characteristic zero. Let $f(x)$ be a polynomial in $k\left[x_{1}, \ldots, x_{m}\right]$, or more generally a definable function in the valued field variables and with values in the valued field with coefficients in $k$ (in the valued field sort and in the residue field sort) in the language $\mathcal{L}_{\text {Pas }}$, and let $\varphi$ be an $\mathcal{L}_{\text {Pas }}$-formula with coefficients in $k$ in the valued field sort and in the residue field sort, free variables $x_{1}, \ldots, x_{m}$ running over valued field variables and no other free variables. Consider, for $n \in \mathbf{N} \cup\{\infty\}$, the definable subassignment $h_{\varphi, n}$ of $h_{\mathcal{L}\left(\mathbf{A}_{k}^{m}\right)}$ associated to the formula $\varphi \wedge$ ord $f=n$. The $h_{\varphi, n}$ 's form a definable partition of the definable subassignment of $h_{\mathcal{L}\left(\mathbf{A}_{k}^{m}\right)}$ defined by $\varphi$. Now set

$$
I_{\varphi, f, \operatorname{mot}}(T):=\sum_{n \in \mathbf{N}} \nu\left(h_{\varphi, n}\right) T^{n} .
$$

8.5.1. Theorem. Let $k$ be a finite extension of $\mathbf{Q}$ with ring of integers $\mathcal{O}$. Let $f(x)$ be a polynomial in $k\left[x_{1}, \ldots, x_{m}\right]$, or more generally a definable function in the valued field variables and with values in the valued field with coefficients in $k$ (in the valued field sort and in the residue field sort) in the language $\mathcal{L}_{\text {Pas }}$, and let $\varphi$ be an $\mathcal{L}_{\text {Pas }}$-formula with coefficients in $k$ in the valued field sort and in the residue field sort, free variables $x_{1}, \ldots, x_{m}$ running over valued field variables and no other free variables. Then the series $I_{\varphi, f, \operatorname{mot}}(T)$ is canonically associated to $\varphi$ and $f$, it is a rational function belonging to $\bar{K}_{0}^{v}\left(\operatorname{Mot}_{k, \overline{\mathbf{Q}}}\right)_{\mathrm{loc}, \mathbf{Q}}[[T]]_{\mathrm{rat}}$, and, for almost all closed points $x$ in $\operatorname{Spec} \mathcal{O}$, the equality

$$
\operatorname{Tr}_{\operatorname{Frob}}\left(I_{\varphi, f, \operatorname{mot}}(T)\right)=I_{\varphi, f}(s, x)
$$

holds after setting $T=q_{x}^{-s}$. Furthermore, if $\varphi^{\prime}$ is another $\mathcal{L}_{\text {Pas }}$-formula with coefficients in $k$ in the valued field sort and in the residue field sort, free variables $x_{1}, \ldots, x_{m}$ running over valued field variables and no other free variables, then

(1) $I_{\varphi \vee \varphi^{\prime}, f, \operatorname{mot}}(T)=I_{\varphi, f, \operatorname{mot}}(T)+I_{\varphi^{\prime}, f, \operatorname{mot}}(T)-I_{\varphi \wedge \varphi^{\prime}, f, \operatorname{mot}}(T)$.

(2) If $\varphi \approx \varphi^{\prime}, I_{\varphi, f, \operatorname{mot}}(T)=I_{\varphi^{\prime}, f, \operatorname{mot}}(T)$.

Proof. The rationality of $I_{\varphi, f, \operatorname{mot}}(T)$ follows from Theorem 7.1.1 and the fact that it satisfies the required specialization property follows from Theorem 8.3.1 Assertions (1) and (2) just follow from 6.1.3 (2) and (3).

\section{Arithmetic Poincaré series versus geometric Poincaré series}

9.1. The geometric Poincaré series $P_{\text {geom }}$. Let $k$ be a field of characteristic 0 and let $X$ be a variety over $k$. Let us remind the reader that we denote by $\pi_{n}$ the morphism of truncation of arcs,

$$
\pi_{n}: \mathcal{L}(X) \longrightarrow \mathcal{L}_{n}(X) .
$$

In the paper [12], we considered the Poincaré series

$$
P_{\text {geom }, X}(T):=\sum_{n \in \mathbf{N}}\left[\pi_{n}(\mathcal{L}(X))\right] T^{n},
$$


and, more generally, for $W$ closed in $X$, the series

$$
P_{\text {geom }, X, W}(T):=\sum_{n \in \mathbf{N}}\left[\pi_{n}\left(\mathcal{L}(X) \cap \pi_{0}^{-1}(W)\right)\right] T^{n} .
$$

One of our main results was the following rationality statement:

9.1.1. Theorem. The series $P_{\text {geom, } X, W}(T)$, considered as a power series over $K_{0}\left(\mathrm{Sch}_{k}\right)_{\text {loc }}$, belongs to the subring of $K_{0}\left(\mathrm{Sch}_{k}\right)_{\text {loc }}[[T]]$ generated by $K_{0}\left(\mathrm{Sch}_{k}\right)_{\text {loc }}[T]$ and the series $\left(1-\mathbf{L}^{a} T^{b}\right)^{-1}$ with $a \in \mathbf{Z}$ and $b$ in $\mathbf{N} \backslash\{0\}$.

9.2. The arithmetic Poincaré series $P_{\text {ar }}$. Let $X$ be a variety defined over a field $k$ of characteristic 0 . Let $W$ be a closed subvariety of $X$ defined over $k$. Now we set

$$
P_{\mathrm{ar}, X, W}(T):=\sum_{n \in \mathbf{N}} \chi_{c}\left(\pi_{n}\left(h_{\mathcal{L}(X)} \cap \pi_{0}^{-1}\left(h_{W}\right)\right)\right) T^{n}
$$

in $K_{0}^{v}\left(\operatorname{Mot}_{k, \overline{\mathbf{Q}}}\right) \mathbf{Q}[[T]]$.

9.2.1. Theorem. Let $h$ be a definable subassignment of $h_{\mathcal{L}(X)}$. Then the series

$$
\sum_{n \in \mathbf{N}} \chi_{c}\left(\pi_{n}(h)\right) T^{n}
$$

belongs to $K_{0}^{v}\left(\operatorname{Mot}_{k, \overline{\mathbf{Q}}}\right)_{\text {loc, } \mathbf{Q}}[[T]]_{\mathrm{rat}}$. In particular, the series $P_{\mathrm{ar}, X, W}(T)$ belongs to $K_{0}^{v}\left(\operatorname{Mot}_{k, \overline{\mathbf{Q}}}\right)_{\text {loc }, \mathbf{Q}}[[T]]_{\text {rat }}$.

Proof. One reduces to the case where $X$ is a closed subvariety of $\mathbf{A}_{k}^{m}$. Then the result follows from Theorem [7.2.1, since

$$
\chi_{c}\left(\pi_{n, X}(h)\right) \mathbf{L}^{-(n+1) m}=\tilde{\nu}_{\mathcal{L}\left(\mathbf{A}_{k}^{m}\right)}\left(\pi_{n, \mathbf{A}_{k}^{m}}^{-1}\left(\pi_{n, X}(h)\right)\right)
$$

and the subassignments $\pi_{n, \mathbf{A}_{k}^{m}}^{-1}\left(\pi_{n, X}(h)\right)$ are stable definable subassignments of $h_{\mathcal{L}\left(\mathbf{A}_{k}^{m}\right)}$ depending on the parameter $n \in \mathbf{N}$. Here $\pi_{n, X}$ and $\pi_{n, \mathbf{A}_{k}^{m}}$ denote the truncation morphisms associated to $X$ and $\mathbf{A}_{k}^{m}$, respectively.

Now assume $k$ is a finite extension of $\mathbf{Q}$ with ring of integers $\mathcal{O}$. Let $R=\mathcal{O}\left[\frac{1}{N}\right]$ with $N$ a nonzero integer. Let $\mathcal{X}$ be a variety over $R$ and let $\mathcal{W}$ be a subvariety. We set $X:=\mathcal{X} \otimes k$ and $W:=\mathcal{W} \otimes k$ and denote by $d$ the dimension of $X$. For $x$ a closed point of $\operatorname{Spec} R$, and $n \in \mathbf{N}$, we consider the canonical morphisms

$$
\pi_{n}: \mathcal{X}\left(\mathcal{O}_{K_{x}}\right) \longrightarrow \mathcal{X}\left(\mathcal{O}_{K_{x}} / \pi_{x}^{n+1} \mathcal{O}_{K_{x}}\right),
$$

where $\pi_{x}$ is a uniformizing parameter of $\mathcal{O}_{K_{x}}$, and we denote by $N_{n, x}(\mathcal{X}, \mathcal{W})$ the cardinality of the finite set $\pi_{n}\left(\mathcal{X}\left(\mathcal{O}_{K_{x}}\right) \cap \pi_{0}^{-1}\left(\mathcal{W}\left(\mathbf{F}_{x}\right)\right)\right)$. By [7] the Poincaré series

$$
P_{x, \mathcal{X}, \mathcal{W}}(T):=\sum_{n \in \mathbf{N}} N_{n, x}(\mathcal{X}, \mathcal{W}) T^{n}
$$

is the expansion of a rational function in $\mathbf{Q}(T)$.

9.2.2. Theorem. Let $k$ be a finite extension of $\mathbf{Q}$ with ring of integers $\mathcal{O}$ and $R=\mathcal{O}\left[\frac{1}{N}\right]$, for some nonzero integer $N$. Let $\mathcal{X}$ be a variety over $R$ and let $\mathcal{W}$ be a subvariety. Then there exists a nonzero multiple $N^{\prime}$ of $N$, such that, for every closed point $x$ of $\operatorname{Spec} \mathcal{O}\left[\frac{1}{N^{\prime}}\right]$,

$$
\operatorname{Tr} \operatorname{Frob}_{x}\left(P_{\text {ar }, X, W}(T)\right)=P_{x, \mathcal{X}, \mathcal{W}}(T)
$$

in $\mathbf{Q}(T)$. 
Proof. We may assume $\mathcal{X}$ is a closed subvariety of $\mathbf{A}_{R}^{m}$ defined by $f_{i}=0,1 \leq i \leq r$, and that $\mathcal{W}$ is defined by $g_{i}=0,1 \leq i \leq s, h_{i} \neq 0,1 \leq i \leq t$, with $f_{i}, g_{i}, h_{i}$ in $R\left[x_{1}, \ldots, x_{m}\right]$. We may also assume that $N$ is a multiple of the discriminant of $k$.

Consider the following formula $\varphi$ in $\mathcal{L}_{\text {Pas }}$ in the $m+1$ free variables $x=$ $\left(x_{1}, \ldots, x_{m}\right)$ and $w$ over the valued field sort:

$$
\begin{aligned}
\bigwedge_{i}\left(\operatorname{ord}\left(g_{i}(x)\right)>0\right) & \wedge \bigwedge_{i}\left(\operatorname{ord}\left(h_{i}(x)\right)=0\right) \\
& \wedge \exists\left(y_{1}, \ldots, y_{m}\right)\left(\bigwedge_{i}\left(\operatorname{ord}\left(x_{i}-y_{i}\right) \geq \operatorname{ord}(w)\right) \wedge \bigwedge_{i}\left(f_{i}(y)=0\right)\right) .
\end{aligned}
$$

For every closed point $x$ in $\operatorname{Spec} R$, the equality

$$
P_{x, \mathcal{X}, \mathcal{W}}\left(q_{x}^{-m-1} q_{x}^{-s}\right)=\frac{q_{x}}{q_{x}-1} I_{\varphi, w}(s, x)
$$

holds, as a direct calculation shows (cf. Lemma 3.1 of [7]). Similarly, the equality

$$
P_{\mathrm{ar}, X, W}\left(\mathbf{L}^{-m-1} T\right)=\frac{\mathbf{L}}{\mathbf{L}-1} I_{\varphi, w, \operatorname{mot}}(T)
$$

follows from the very definitions, hence the result is a direct consequence of Theorem 8.5.1,

\section{An example: Branches of plane curves}

10.1. Let $k$ be a field of characteristic zero. Consider a formal branch of a plane curve

$$
X:\left\{\begin{array}{l}
x=w^{m}, \\
y=\sum_{j \geq m} a_{j} w^{j},
\end{array}\right.
$$

with coefficients $a_{j}$ in $k$. We can assume that $\operatorname{gcd}\left\{j \mid a_{j} \neq 0\right\}=1$. We define $e_{0}=m, \beta_{1}=\inf \left\{j \mid a_{j} \neq 0\right.$ and $\left.e_{0} \nmid j\right\}, e_{1}=\left(e_{0}, \beta_{1}\right)$, and, by induction, $\beta_{i}=$ $\inf \left\{j \mid a_{j} \neq 0\right.$ and $\left.e_{i-1} \nmid j\right\}, e_{i}=\left(e_{i-1}, \beta_{i}\right)$. The sequence of the integers $e_{i}$ being strictly decreasing, for some smallest integer $g, e_{g}=1$. We also define $n_{i}$, for $1 \leq i \leq g$, by $e_{i-1}=n_{i} e_{i}$, and set $N_{i}:=\prod_{1 \leq j \leq i} n_{j}$. We set $\beta_{0}=m, \beta_{g+1}=\{\infty\}$ and $N_{0}=1$.

For any integer $r \geq 1$, we denote by $\mu(r)$ the group of $r$-th roots of unity in $\mathbf{C}$. The following well-known lemma is stated for convenience.

10.1.1. Lemma. Let $\zeta$ be an $m$-th root of unity. Then $\operatorname{ord}_{w}(y(w)-y(\zeta w))=\beta_{i}$ if and only if $\zeta \in \mu\left(e_{i-1}\right) \backslash \mu\left(e_{i}\right)$, for $i \geq 1$.

10.2. The definition of $\mathcal{L}(X), \mathcal{L}_{n}(X)$ and $\pi_{n}$ is extended in a straightforward way to formal varieties to set $\mathcal{X}_{n}:=\pi_{n}\left(\mathcal{L}_{n}(X) \cap \pi_{0}^{-1}(0)\right)$. We define the Poincaré series

$$
P_{\text {geom }, X, 0}(T):=\sum_{n \geq 0}\left[\mathcal{X}_{n}\right] T^{n} .
$$

When $X$ is an analytically irreducible algebraic curve this definition coincides with the one given in 9.1

To define $P_{\text {ar }}$ in this situation we consider the formula $\varphi_{n}$ in the free variables $x_{1}, \ldots, x_{n}$ and $y_{1}, \ldots, y_{n}$

$$
\exists w\left(\operatorname{ord}_{t}\left(x-w^{m}\right)>n \wedge \operatorname{ord}_{t}\left(y-\sum_{m \leq j \leq n} a_{j} w^{j}\right)>n\right),
$$


with $x=\sum_{1 \leq i \leq n} x_{i} t^{i}, y=\sum_{1 \leq i \leq n} y_{i} t^{i}$ and $w=\sum_{1 \leq i \leq n} w_{i} t^{i}$, and set

$$
P_{\text {ar, } X, 0}(T)=\sum_{n \geq 0} \chi_{c}\left(\varphi_{n}\right) T^{n}
$$

When $X$ is an analytically irreducible algebraic curve this definition coincides with the one given in 9.2

10.2.1. Proposition. Let $k$ be a field of characteristic zero. Consider a formal branch of a plane curve

$$
X:\left\{\begin{array}{l}
x=w^{m} \\
y=\sum_{j \geq m} a_{j} w^{j},
\end{array}\right.
$$

with coefficients $a_{j}$ in $k$. Assume $k$ contains all $m$-th roots of unity. Then

$$
P_{\text {geom }, X, 0}(T)=\frac{1}{1-T}+\frac{\mathbf{L}-1}{1-\mathbf{L} T} \frac{T^{m}}{1-T^{m}}
$$

and

$$
P_{\mathrm{ar}, X, 0}(T)=\frac{1}{1-T}+\frac{\mathbf{L}-1}{1-\mathbf{L} T}\left[\frac{1}{m} \frac{T^{m}}{1-T^{m}}+\sum_{1 \leq i \leq g} \frac{N_{i}-N_{i-1}}{m} \frac{\mathbf{L}^{\beta_{i}-m} T^{\beta_{i}}}{1-\mathbf{L}^{\beta_{i}-m} T^{\beta_{i}}}\right] .
$$

In particular, the poles of the rational function $P_{\mathrm{ar}, X, 0}(T)$ of the form $T=\mathbf{L}^{\alpha}, \alpha$ in $\mathbf{Q} \backslash\{0,-1\}$, are exactly $T=\mathbf{L}^{\frac{m}{\beta_{i}}-1}, 1 \leq i \leq g$.

Proof. For every integer $\ell$ such that $0<\ell \leq \frac{n}{m}$, we set $\mathcal{X}_{n, \ell}=\mathcal{X}_{n} \cap\left\{(x, y) \mid \operatorname{ord}_{t} x=\right.$ $\ell m\}$, with $x=\sum_{1 \leq i \leq n} x_{i} t^{i}$ and $y=\sum_{1 \leq i \leq n} y_{i} t^{i}$. Similarly, we consider the formula $\varphi_{n, \ell}=\varphi_{n} \wedge\left(\operatorname{ord}_{t} x=\ell m\right)$. So we have $\left[\mathcal{X}_{n}\right]=1+\sum_{0<\ell \leq \frac{n}{m}}\left[\mathcal{X}_{n, \ell}\right]$ and $\chi_{c}\left(\varphi_{n}\right)=$ $1+\sum_{0<\ell \leq \frac{n}{m}} \chi_{c}\left(\varphi_{n, \ell}\right)$, and the result follows directly from the next lemma.

10.2.2. Lemma. Fix an integer $\ell \leq \frac{n}{m}$. Let $i$ be the unique integer $0 \leq i \leq g$ such that $\frac{n}{\beta_{i+1}}<\ell \leq \frac{n}{\beta_{i}}$. Then

$$
\left[\mathcal{X}_{n, \ell}\right]=(\mathbf{L}-1) \mathbf{L}^{n-\ell m} \quad \text { and } \quad \chi_{c}\left(\varphi_{n, \ell}\right)=\frac{N_{i}}{m}(\mathbf{L}-1) \mathbf{L}^{n-\ell m} .
$$

Proof. We consider the variety $W=\mathbf{G}_{m, k} \times \mathbf{A}_{k}^{n-\ell m}$ with coordinate $w_{\ell}$ on the first factor and $\left(w_{\ell+1}, \ldots, w_{n-\ell m+\ell}\right)$ on the second factor. There is a morphism $h: W \rightarrow \mathbf{A}_{k}^{n} \times \mathbf{A}_{k}^{n}$ sending a point $\left(w_{\ell}, \ldots, w_{n-\ell m+\ell}\right)$ to the first $n$ coefficients of the series $w^{m}$ and $\sum_{j \ell \leq n} a_{j} w^{j}$. Let $Z$ be the image of $W$ by $h$. It is a locally closed subvariety of $\mathbf{A}_{k}^{n} \times \mathbf{A}_{k}^{n}$. It follows from Lemma 10.1.1 that $h: W \rightarrow Z$ is a Galois cover with group $G=\mu\left(e_{i}\right)$ acting by multiplication on $W$. By construction, $\chi_{c}\left(\varphi_{n, \ell}\right)$ is equal to $\chi_{c}(W, \delta)$, with $\delta$ the central function on $G$ which takes value 1 at the identity and zero elsewhere. But now remark that $\chi_{c}(W, \alpha)=0$ for $\alpha$ a nontrivial irreducible character of $G$ and that $\chi_{c}(W, \alpha)=(\mathbf{L}-1) \mathbf{L}^{n-\ell m}$ when $\alpha$ is the trivial character (cf. Lemma 1.4.3 of [11]). The second equality follows, since, when $\delta$ is expressed as a $\mathbf{Q}$-linear combination of irreducible characters, the coefficient of the trivial character is $e_{i}^{-1}=\frac{N_{i}}{m}$. For the first equality it is enough to remark that $Z=\mathcal{X}_{n, \ell}$ and that $\left[W / \mu\left(e_{i}\right)\right]=(\mathbf{L}-1) \mathbf{L}^{n-\ell m}$.

10.2.3. Remark. The calculation of the $p$-adic Poincaré series for branches of plane curves was carried out in [3]. 


\section{REFERENCES}

1. J. Ax, The elementary theory of finite fields, Ann. of Math., 88 (1968), 239-271. MR 37:5187

2. S. del Baño Rollin, V. Navarro Aznar, On the motive of a quotient variety, Collect. Math., 49 (1998), 203-226. MR 2000d:14009

3. D. Bollaerts, On the Poincaré series associated to the p-adic points on a curve, Acta Arith., 51 (1988), 9-30. MR 89i:11130

4. Z. Chatzidakis, L. van den Dries, A. Macintyre, Definable sets over finite fields, J. Reine Angew. Math., 427 (1992), 107-135. MR 94c:03049

5. R. Cluckers, D. Haskell, The Grothendieck ring of the p-adic numbers, preprint (5 pages).

6. P. Deligne, La conjecture de Weil. I, Inst. Hautes Études Sci. Publ. Math., 43 (1974), 273-307. MR 49:5013

7. J. Denef, The rationality of the Poincaré series associated to the p-adic points on a variety, Invent. Math., 77 (1984), 1-23. MR 86c:11043

8. J. Denef, p-adic semi-algebraic sets and cell decomposition, J. Reine Angew. Math., 369 (1986), 154-166. MR 88d:11030

9. J. Denef, On the evaluation of certain p-adic integrals, Séminaire de théorie des nombres, Paris 1983-84, 25-47, Progr. Math., 59, Birkhäuser Boston, Boston, Mass., 1985. MR 88j:11031

10. J. Denef, On the degree of Igusa's local zeta function, Amer. J. Math., 109 (1987), 991-1008. MR 89d:11108

11. J. Denef, F. Loeser, Motivic Igusa zeta functions, J. Algebraic Geom., 7 (1998), 505-537. MR 99j:14021

12. J. Denef, F. Loeser, Germs of arcs on singular algebraic varieties and motivic integration, Invent. Math., 135 (1999), 201-232. MR 99k:14002

13. J. Denef, F. Loeser, Motivic exponential integrals and a motivic Thom-Sebastiani Theorem, Duke Math. J., 99 (1999), 285-309. MR 2000k:14006

14. J. Denef, F. Loeser, Motivic integration, quotient singularities and the McKay correspondence, preprint February 1999.

15. H. Enderton, A mathematical introduction to logic, Academic Press (1972), New York-London. MR 49:2239

16. M. Fried, D. Haran, M. Jarden, Galois stratifications over Frobenius fields, Adv. in Math., 51 (1984), 1-35. MR 86c:12007

17. M. Fried, D. Haran, M. Jarden, Effective counting of the points of definable sets over finite fields, Israel J. Math., 85 (1994), 103-133. MR 95k:12016

18. M. Fried, M. Jarden, Field arithmetic, Ergebnisse der Mathematik und ihrer Grenzgebiete, 3. Folge Band 11 (1986), Springer-Verlag, Berlin, Heidelberg. MR 89b:12010

19. M. Fried, G. Sacerdote, Solving diophantine problems over all residue class fields of a number field and all finite fields, Ann. of Math., 100 (1976), 203-233. MR 58:10722

20. H. Gillet, C. Soulé, Descent, motives and K-theory, J. Reine Angew. Math., 478 (1996), 127-176. MR 98d:14012

21. F. Guillén, V. Navarro Aznar, Un critère d'extension d'un foncteur défini sur les schémas lisses, preprint (1995), revised (1996).

22. C. Kiefe, Sets definable over finite fields, their zeta-functions, Trans. Amer. Math. Soc., 223 (1976), 45-59. MR 54:10272

23. S. Lang, A. Weil, Number of points of varieties in finite fields, Amer. J. Math., 76 (1954), 819-827. MR 16:398d

24. A. Macintyre, Rationality of p-adic Poincaré series: Uniformity in p, Ann. Pure Appl. Logic, 49 (1990), 31-74. MR 92b:11085

25. J. Oesterlé, Réduction modulo $p^{n}$ des sous-ensembles analytiques fermés de $\mathbf{Z}_{p}^{N}$, Invent. Math., 66 (1982), 325-341. MR 83j:12014

26. J. Pas, Uniform p-adic cell decomposition and local zeta functions, J. Reine Angew. Math., 399 (1989), 137-172. MR 91g:11142

27. M. Presburger, Uber die Vollständigkeit eines gewissen Systems des Arithmetik ... , Comptesrendus du I Congrès des Mathématiciens des Pays Slaves, Warsaw (1929), 92-101.

28. A. Scholl, Classical motives, in Motives, U. Jannsen, S. Kleiman, J.-P. Serre, Eds., Proceedings of Symposia in Pure Mathematics, Volume 55 Part 1 (1994), 163-187. MR 95b:11060

29. J.-P. Serre, Zeta and L functions, Arithmetical Algebraic Geometry, Proc. Conf. Purdue Univ., Harper \& Row, New York (1965), 82-92. MR 33:2606 
30. J.-P. Serre, Quelques applications du théorème de densité de Chebotarev, Inst. Hautes Études Sci. Publ. Math., 54 (1981), 323-401. MR 83k:12011

31. W. Veys, Reduction modulo $p^{n}$ of p-adic subanalytic sets, Math. Proc. Cambridge Philos. Soc., 112 (1992), 483-486. MR 93i:11142

Department of Mathematics, University of Leuven, Celestijnenlaan 200B, 3001 LeuVEN, Belgium

E-mail address: Jan.Denef@wis.kuleuven.ac.be

$U R L:$ http://www.wis.kuleuven.ac.be/wis/algebra/denef.html

Département de mathématiques et applications, École Normale Supérieure, 45 rue D'Ulm, 75230 Paris Cedex 05, France (UMR 8553 du CNRS)

E-mail address: Francois.Loeser@ens.fr

$U R L:$ http://www.dma.ens.fr/ loeser/ 